\title{
ENQUÊTES SUR LE PLANCHER SALARIAL ET LA RÉMUNÉRATION DES ENSEIGNANTS : UN ÉTAT DE L'ART - 2008-2021
}

\section{ARTICLE ORIGINAL}

LIMA, Maria José Rocha ${ }^{1}$, RAMOS, Fernando Sadio²

LIMA, Maria José Rocha. RAMOS, Fernando Sadio. Enquêtes sur le plancher salarial et la rémunération des enseignants : un état de l'art - 2008-2021. Revista Científica Multidisciplinar Núcleo do Conhecimento. Année. 06, Ed. 11, vol. 08, p. 100 à 133. Novembre 2021. ISSN : 2448-0959, Lien d'accès : https://www.nucleodoconhecimento.com.br/education-fr/remuneration-desenseignants, $\quad$ DOI: $\quad 10.32749$ /nucleodoconhecimento.com.br/education$\mathrm{fr} /$ remuneration-des-enseignants

\section{RÉSUMÉ}

Cet article présente une enquête sur les études sur les salaires planchers et la rémunération des enseignants entre 2008 et 2021[3]. En ce sens, la revue de la littérature sur le plancher salarial du magistère est une pièce fondamentale. À cette fin, la méthodologie développée pour la recherche bibliographique est basée sur I'utilisation scientifique et prudente de la théorie de la Evidence-Based Practice (EBP)- en portugais, Práticas Baseadas em Evidências (PBE). C'est un état de l'art qui élimine l'un des facteurs les plus décisifs pour la transformation de la réalité éducative: les études sur les salaires et la rémunération des enseignants brésiliens. Dans cette étude, une autre contradiction entre les discours emphatiques

\footnotetext{
${ }^{1}$ Étudiant au doctorat en éducation à l'Université Internationale Ibéroaméricaine - UNINI - ; Master en éducation de l'Université fédérale de Bahia-UFBA-, Psychopédagogie de I'Université Cândido Mendes - AVM, Spécialiste en psychanalyse de l'Association brésilienne d'études et de recherche en psychanalyse - ABEPP-, Spécialiste des cultures noires dans I'Atlantique de l'Université de Brasília - UnB-, Spécialiste en méthodologie d'enseignement de l'enseignement supérieur, Faculté d'éducation de Bahia - FEBA - Diplôme complet de formation spéciale au programme d'études secondaires, dans le domaine des sciences de la santé ; Enseignant primaire, diplômé de l'Instituto Central de Educação Isaías Alves. ORCID ID: 0000-0003-1766-2169 ${ }^{2}$ Conseiller. Docteur en sciences sociales/sciences de l'éducation, programme d'études, Profesorado e Instituciones Educativas, Université de Grenade, Espagne. Master en philosophie contemporaine de l'Université de Coimbra, Portugal. Diplôme en philosophie de l'Université de Coimbra, Portugal. Directeur de DEDICA Magazine de l'éducation et des sciences humaines. (0000-0001-7654-5638) - ORCID
}

RC: 101662

Disponible en: https://www.nucleodoconhecimento.com.br/education-fr/remunerationdes-enseignants 
ou grandilo chauds en défense de la valorisation de l'enseignement et les pratiques des autorités politiques et éducatives est exposée, nous appelant également la faible production de monographies, de mémoires et de thèses de doctorat, dans le monde universitaire, sur le plancher salarial et la rémunération des enseignants. Même avec la proclamation de l'appréciation de l'enseignant comme un facteur décisif pour assurer la qualité de l'éducation, plus de dix ans se sont écoulés depuis la création du salaire plancher, cependant, environ $60 \%$ des municipalités brésiliennes ne l'ont pas encore mis en œuvre et cela n'a pas la répercussion attendue dans les études universitaires.

Mots-clés : État de l'art, Salaire plancher, Salaire de l'enseignant, Salaire de l'enseignant, Professionnalisation de l'enseignant.

\section{INTRODUCTION}

Au Brésil, malheureusement en raison d'une tradition culturelle inique, la garantie de l'éducation en tant que droit social a été marquée par un retard séculaire et une lenteur impensable. Les causes de ce retard éducatif ont été soulignées par des théoriciens, tels que le sociologue Florestan Fernandes (1989, p. 160), qui, en analysant l'histoire brésilienne, identifie la dépendance économique, l'obscurantisme des classes dominantes et un conflit permanent pris à l'intérieur de l'école, qui aide peu à résoudre les problèmes, comme facteurs de retard. C'est comme une mesure ininterrompue de la corrélation des forces. Ainsi, il y a des conflits de projet scolaire, de projet de valorisation, ou non, de l'enseignant ; projet de société; le projet de I'homme, enfin, comme une lutte politique incessante. Ce qu'un gouvernement fait, un autre le défait. Quels sont ces intérêts qui font bouger la non-application de la loi qui a institué le plancher salarial? Les différends dont Florestan Fernandes nous parle, parmi les sceptiques de droite ou de gauche qui sabotent presque toujours l'éducation au Brésil, seraient-ils exprimés?

En 1932, le Manifeste des Pionniers de la Nouvelle Éducation, qui comptait parmi ses abonnés les plus éminents Fernandes de Azevedo et Anísio Teixeira, commence 
le document appelant à ce que dans la hiérarchie des problèmes nationaux, aucun ne soit dépassé par l'éducation, pas même ceux à caractère économique. Le célèbre penseur de l'éducation Anísio Teixeira a averti que "sans éducation, la démocratie serait un vain mot utilisé pour justifier la triste farce d'un suffrage universel dérisoire " (LIMA, 2011, p. 66). Pour Florestan, « un point essentiel est ce qui concerne la tradition culturelle et ce qu'elle représente de la limitation culturelle de l'enseignant, moins en théorie qu'en pratique » (FERNANDES, 1898, p. 157).

La vision du magistère comme sacerdoce s'est croisée et consolidée à travers les siècles. Cette conception religieuse et traditionnelle dans la culture brésilienne, bien construite dans les premiers jours de la colonisation, lorsqu'elle était dirigée par des religieux, était l'un des graves problèmes rencontrés par les enseignants pour leur professionnalisation et l'exécution du travail scolaire professionnel. Dans cette tradition, il était, et est, inacceptable pour l'enseignant de discuter de la rémunération; le maître doit prendre le magistère comme une mission: être idéaliste, être pauvre et heureux dans l'exercice de la profession. II n'est pas rare que l'enseignant n'ait même pas l'argent pour acheter des livres.

En 1827, l'empereur Dom Pedro I a accordé la loi des écoles de premières lettres du 15 octobre 1827, établissant à l'article 3 la rémunération des enseignants, un plancher salarial jamais mis en œuvre. Son successeur, Don Pedro II, a même déclaré que " s'il n'était pas empereur, il voudrait être enseignant " et qu'il ne connaissait pas « une mission plus grande et plus noble que celle de diriger la jeune intelligence et de préparer les hommes de l'avenir ». Le dernier monarque de l'Empire du Brésil a régné pendant près de 50 ans et rien de concret n'a fait pour la rémunération et la reconnaissance de l'enseignant.

Les belles et expressives paroles prononcées et les actes législatifs des empereurs du Brésil, de 1827 à 1889, n'ont pas eu les répercussions annoncées dans la politique de rémunération des enseignants. Malgré la non-mise en œuvre du sol du magistère, l'acte de créer le sol en 1827 était emblématique. Tout cela constitue la 
preuve la plus éloquente de ce décalage entre discours et pratique dans l'histoire politique de la rémunération des enseignants dans les écoles publiques brésiliennes.

Les autorités brésiliennes parlent avec éloquence de l'importance de l'enseignant, cependant,

cheios de meras frases de efeito, demagógicas, insinceras, ou discursos oficiais apenas ressaltam no magistério o seu caráter de sacerdócio (que realmente), mas sem a contrapartida de oferecer as condições necessárias a que o professor possa atuar na plenitude dos seus recursos e das suas qualificações (TEIXEIRA, 1999, p. 9).

Ces discours, qui sont souvent transmis, du point de vue du magistère comme une mission à caractère religieux et/ou assistance niste/paternaliste semblent fonctionner, en règle générale, empêchant les éducateurs, les universitaires, et même les syndicalistes, d'aborder et de défendre les salaires, le plancher salarial, la rémunération décente de l'enseignement pour le magistère, de sorte que quand ils le font, c'est avec une certaine tibieza.

L'idée de "l'enseignant vocationnel », une vision purement subjective, a un impact négatif sur la tâche pédagogique, constituant un obstacle pour surmonter l'idée erronée selon laquelle pour enseigner, certaines compétences sont suffisantes, sans tenir compte de la complexité de la tâche. Enseigner n'est pas un travail pour les laïcs. La didactique et la pédagogie sont des domaines scientifiques avec une accumulation importante de connaissances.

Ainsi, deux défis sérieux doivent être relevés pour l'exécution du travail scolaire professionnel et l'augmentation de la qualité de l'éducation: la non-reconnaissance de l'enseignement en tant que profession et l'incertitude quant à l'objectif de l'école. Afin de surmonter ces deux problèmes, il faut faire un mouvement fort qui s'oppose à la situation impensable que l'enseignant reçoive un salaire plancher presque toujours bien inférieur aux salaires reçus par les professionnels ayant le même niveau de formation. Cette situation l'oblige à travailler dans plusieurs écoles en même temps et à accepter toujours l'exécution de travaux scolaires dans des espaces inadéquats. 
Les enseignants travaillent dans les conditions les plus précaires : à côté des toilettes fétides, dans des écoles avec des salles divisées même par des tabiques, sans eau, sans salon. Ils fonctionnent le plus souvent sans équipement de base, tel que portefeuilles, bureaux, armoires, entre autres nécessaires. Dans les endroits éloignés, inhospitaliers et non sécurisés, les agressions et les agressions contre les enseignants à l'intérieur des écoles sont courantes.

En 2020, 4300 écoles publiques n'avaient pas de toilettes et l'Internet à large bande n'atteignait pas 17200 (20,5\%) en 2020. En outre, 35800 écoles continuent de ne pas être enseignées, soit $26,6 \%$ du total, selon le recensement scolaire de 2019 . En ce qui concerne les ressources didactiques et pédagogiques, les enseignants, parce qu'ils n'en ont pas, sont en concurrence déloyale avec les ressources médiatiques, comme nous le voyons en cette période de pandémie. Le Covid-19 a exposé la situation tragique des enseignants " crachés et craies ", sans cartes, globes, illustrations, au 21 ème siècle et à l'ère de la révolution technologique.

Il est également essentiel, pour la résolution de ces problèmes, la définition claire du but de l'école. Pour cela, il est nécessaire de stimuler et d'élever la production scientifique et un mouvement qui favorise le dépassement de cette confusion établie par rapport à la fonction de l'école: lieu de protection, abri, dépôt pour les enfants ou espace unique pour effectuer des travaux scolaires, qui doit remplir son objectif principal d'offrir les connaissances et les contenus techniques et scientifiques nécessaires à la survie symbolique.

L'un des discours les plus répandus pour la déconstruction de cette vision de l'enseignant-prêtre a été prononcé par le professeur de l'Université de Bahia - UFBA, le journaliste et écrivain João Carlos Teixeira, à l'Assemblée législative de Bahia, en 1999, quand il a dit:

Por lamentável (e iníqua) tradição, no Brasil o professor costuma ser simultaneamente mártir e herói, quando deveria ser apenas (sobretudo) uma pessoa capacitada para o exercício da sua relevante missão social, prestigiada pela sociedade e amparada pelos poderes públicos, com remuneração à altura da importância de

RC: 101662

Disponible en: https://www.nucleodoconhecimento.com.br/education-fr/remunerationdes-enseignants 
que o seu trabalho se reveste para toda a coletividade (TEIXEIRA, 1999, p. 9).

Ce n'est qu'au 21e siècle que le plancher salarial professionnel national a été établi par la loi. C'est ainsi que le 16 juillet 2008, le Président de la République de l'époque, Luiz Inácio Lula da Silva, a sanctionné la loi 11.738/2008. Cette loi a établi le plancher salarial professionnel national pour les professionnels de l'éducation publique de base - PSPN - établissant une valeur à ajuster obligatoirement chaque année et «en dessous de laquelle l'Union, les États, le District fédéral et les municipalités ne seront pas en mesure de fixer le salaire initial des carrières de l'enseignement public de l'enseignement de base, pour le voyage de, maximum de 40 (quarante) heures par semaine »(BRASIL, 2008). Pourtant, même après cette attente laïque, plus de la moitié des 5570 municipalités du Brésil ne s'y conforment pas.

Compte tenu de ce scénario, et selon la proposition, nous avons sélectionné les dix auteurs qui ont le plus publié sur le plancher salarial de l'enseignement public de l'éducation de base et avons cherché à appréhender les résultats les plus mis en évidence par ces auteurs en ce qui concerne la mise en œuvre du PSPN, créé par la loi 11738 , du 16 juillet 2008.

Les gens, les autorités éducatives, les entrepreneurs et surtout les politiciens font des discours éloquents et adjectifs de reconnaissance à l'enseignant, mais la pratique est manifestement contradictoire.

\section{SALAIRE PLANCHER ET RÉMUNÉRATION DES ENSEIGNANTS : UNE REVUE DE LA LITTÉRATURE}

Tel que contextualisé précédemment, l'objet de ce travail est la mise en œuvre du plancher salarial des enseignants, sur lequel nous présentons cette revue de la littérature. À cette fin, la méthodologie développée pour la recherche bibliographique est basée sur l'utilisation scientifique et prudente de la théorie de la Evidence-based practice $(E B P)$. 
Il convient de noter ici que la discussion sur les recherches bibliographiques fondées sur des données probantes est née des études de la médecine factuelle (MBE) et s'étend à d'autres disciplines avec le terme EBP. Selon Santos et al. (2007),

a PBE prevê metodologias e processos para a identificação de evidências de que um certo tratamento, ou meio diagnóstico, é efetivo, estratégias para avaliação da qualidade dos estudos e mecanismos para a implementação na assistência. (...) O movimento da PBE teve origem simultânea na McMaster University (Ontario, Canadá) e na University of York (Reino Unido). Evidência é aquilo que é claro, a constatação de uma verdade que não suscita qualquer dúvida. Evidência científica representa uma prova de que um determinado conhecimento é verdadeiro ou falso. Para que se tenha evidência científica é necessário que exista pesquisa prévia, conduzida dentro dos preceitos científicos (SANTOS et al., 2007).

En ce sens, la proposition était d'effectuer une recherche bibliographique basée sur des préceptes scientifiques dans le but d'extraire de l'objet principal « paiement du plancher salarial professionnel national - PSPN », le travail de recherche le plus corrélé et le plus important pour l'étude.

De plus, nous justifions l'adoption de cette méthodologie parce qu'il y a des critiques des revues de littératures traditionnelles, qui n'ont pas de méthodologies spécifiques et explicites, montrant ainsi un caractère aléatoire et biaisé total dans leurs recherches. Santos et al. ( 2007) soulèvent cette discussion lorsqu'elle révèle que :

As revisões de literatura tradicionais (hoje chamadas revisões
narrativas) há muito são criticadas, uma vez que o método de busca
bibliográfica e seleção dos estudos não são padronizados e
explicitados. Os resultados obtidos com tais revisões são
tendenciosos, não esgotam toda a literatura disponível sobre o tema
pesquisado e geralmente são inconclusivos. A busca de evidência
requer adequada definição da pergunta de pesquisa e criação de
estrutura lógica para a busca bibliográfica de evidências na literatura,
que facilitam e maximizam o alcance da pesquisa (SANTOS et al.,
2007).

Selon Bariani et al. (2007), pour les recherches bibliographiques fondées sur des données probantes, certaines lignes directrices devraient être suivies, comme nous l'avons fait dans ce travail : 
I. Aujourd'hui, les recherches documentaires se font en grande partie dans des annuaires en ligne, c'est-à-dire dans des bases de données en ligne. Pour ce travail, nous avons effectué une recherche dans la base de données de Capes, Scientific Electronic Library Online - Scielo, Google Scholar et Google Books.

II. Sélection de descripteurs, ou mots-clés, alignés sur les spécificités du thème. De cette façon, nous évitons les recherches avec des descripteurs larges et généralistes.

III. Délimitation de la typicité des mots-clés, priorisation de l'utilisation des noms et des adjectifs; I'utilisation de mots au singulier et, enfin, éviter les prépositions, les conjonctions ou les articles.

IV. Vérification et validation des mots choisis sur les sites d'édition dans les principaux domaines d'études liés à la recherche.

V. Filtrage de la recherche. La recherche doit être bien délimitée, il y a donc quelques façons qui ont été suivies dans cette recherche:

A) Recherche du matériel dans le laps de temps de la recherche;

B) Recherche exacte des termes. La recherche exacte est facilitée lorsque les mots ou les expressions sont écrits entre guillemets;

C) Utilisation d'opérateurs logiques booléens AND, OR et NOT, où AND est utilisé pour localiser plus d'un terme dans la même référence; NO exclure qu'un terme commun soit trouvé avec le mot-clé utilisé; et OR pour exclure que des termes plus courants soient trouvés avec le mot-clé utilisé.

Tableau 1 : expressions de recherche clés

\section{Plancher salarial AND magistère}

Salaire plancher

AND
PSPN AND magistère

formation PSPN AND formation




\section{professionnelle}

Salaire plancher AND enseignant

Salaire plancher AND enseignant

Salaire plancher AND éducateur

Salaire plancher AND éducation travailleurs

Salaire plancher AND éducation professionnelle

PSPN AND enseignant

PSPN AND enseignant

PSPN AND éducateur

des PSPN AND travailleur de l'éducation

PSPN AND éducation

Source : Élaboration propre (2021).

À partir du thème Plancher salarial professionnel national, les termes clés ont été délimités, à qui sont Plancher salarial et PSPN. Les recherches ont été effectuées avec la coupure du 1er janvier 2008 au 1er janvier 2021 en mode « recherche avancée » sur le portail CAPES, Scielo, Google Scholar et Google Books. Les termes OR et AND ont ensuite été classés en tant qu'opérateurs booléens. La combinaison des termes a été effectuée afin de rechercher dans les bases de données les termes: Salaire plancher ou PSPN, en combinant la recherche avec les synonymes conceptuels sur les enseignants. De plus, il convient de mentionner que les prépositions « da» et «de» ont été supprimées dans le but d'affiner la recherche.

Après la recherche de littérature, une base de données a été créée avec tous les articles, thèses et livres trouvés. Pour l'assemblage de la base de données, les articles répétés n'ont pas été pris en compte, les publications en dehors de la période échantillonnée et qui n'ont pas été publiées en portugais.

Afin d'élargir les connaissances sur la littérature nationale, notre recherche bibliographique était basée sur la recherche d'articles nationaux, de monographies, de thèses, de mémoires et de livres qui abordait, de manière contextualisée, le plancher salarial. Ce filtre a été élaboré à partir du titre et du résumé d'articles scientifiques, de thèses, de dissertations, de monographies et de livres. 
Les descripteurs les plus efficaces étaient " salaire et plancher d'enseignement », " plancher salarial et formation professionnelle » et " plancher salarial et enseignant ». L'autre, " plancher salarial et éducateur », " plancher salarial et travailleur de l'éducation ", " plancher salarial et éducation " à, ensemble, 12 résultats. Finalement, la recherche a totalisé 60 productions bibliographiques à analyser.

Parmi les sources de recherche utilisées, Google Scholar et Google Books sont revenus avec le plus grand volume de données. Par conséquent, le critère de classification de la pertinence du contenu a été utilisé, suivi de la sélection des dix premiers travaux qui ont envisagé les critères et les objectifs de cette recherche. Le délai était du 1er janvier 2008 au 1er janvier 2021, justifié par la date d'entrée en vigueur du parquet et s'étendant jusqu'à la période d'évolution de la loi FUNDEB.

Nous reconnaissons la limitation des critères de recherche, bien que nous comprenions qu'il s'agit d'un effort visant à établir un lien entre la recherche et les discussions alignées sur l'avancement des discussions sur le plancher salarial national. Les articles internationaux, les nouvelles, les productions qui ne traitaient pas du plancher salarial dans le contexte brésilien ou qui l'abordaient de manière marginale n'ont pas été pris en compte.

\section{LA PRODUCTION BRÉSILIENNE ET SES CARACTÉRISTIQUES}

Bien que les approches et la consolidation des politiques d'appréciation des enseignants aient évolué depuis des décennies, c'est à partir de la mi-2010 que la discussion dans le domaine du salaire plancher et de la rémunération des enseignants gagne en importance, ce qui reflète la croissance des publications, tant dans les revues scientifiques que dans les livres spécialisés.

a. Nombre de recherches au fil des ans

Au fil des ans, les discussions sur la loi PSPN ont été centralisées dans la mise en œuvre de la politique. En analysant les productions sur 13 ans, nous avons observé une augmentation des publications dans les années 2016 et 2019, par rapport aux

RC: 101662

Disponible en: https://www.nucleodoconhecimento.com.br/education-fr/remunerationdes-enseignants 
autres années de publications. II y a une augmen tation en 2016, dont la corrélation possible est les élections municipales et les changements qui pourraient provenir des nouvelles administrations municipales. Une autre hausse possible en 2019 semble coïncider avec le mouvement social et l'articulation sur le Fonds national pour le développement de l'éducation - FUNDEB, qui avait, jusqu'à ce moment, un caractère provisoire.

Figure 1 - Nombre de recherches au fil des ans

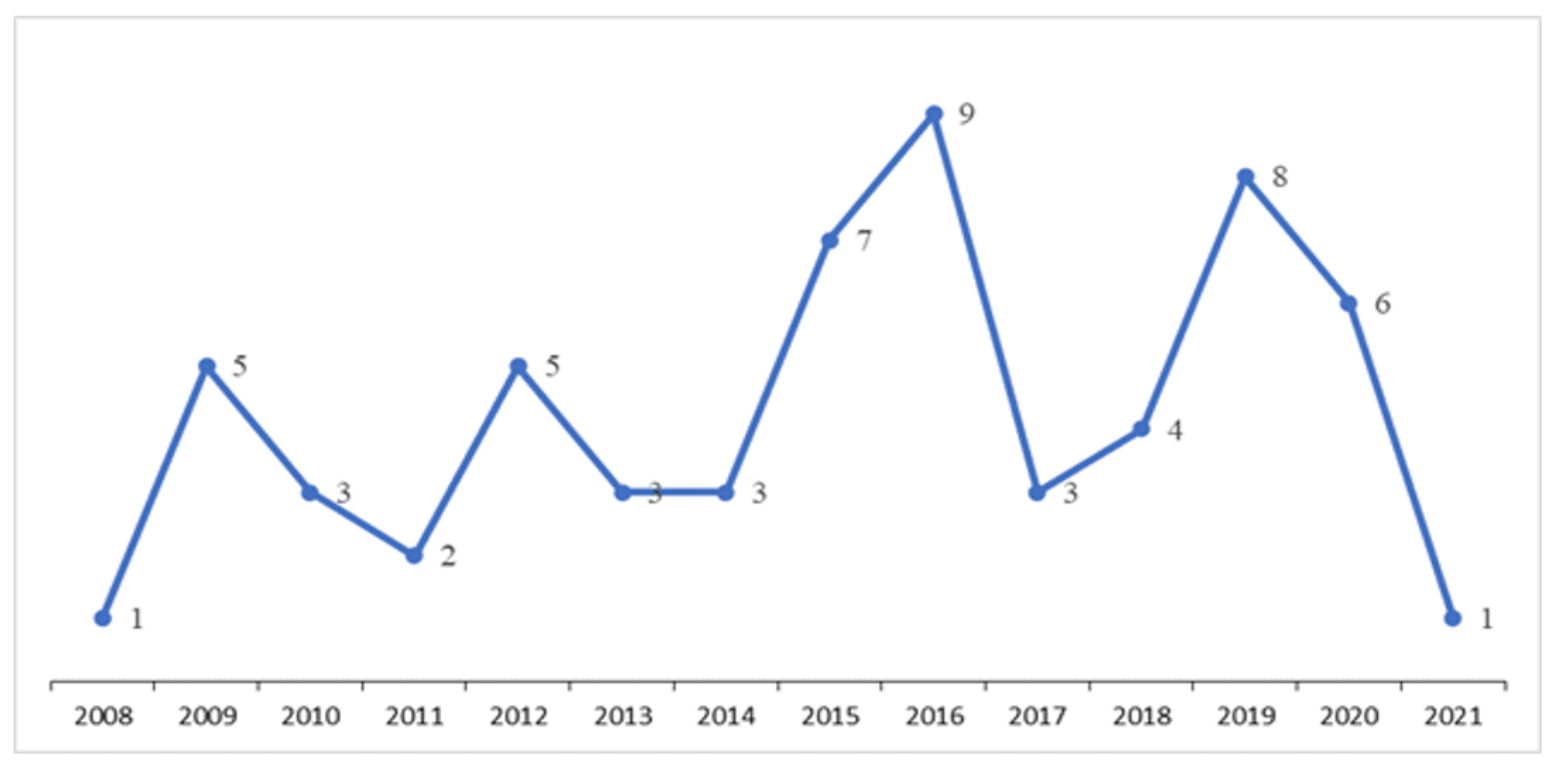

Source : Élaboration propre (2021).

B. Types de parutions

En général, le type de publication prédominant sont les articles et les livres, représentant $86,7 \%$ de l'ensemble total de cette enquête. Ces études ont été principalement menées par des productions dans le domaine de l'éducation. Les thèses, mémoires et monographies avaient une faible représentativité dans l'ensemble de cette enquête par rapport aux articles et aux livres. 
Graphique 2 - Types de parutions

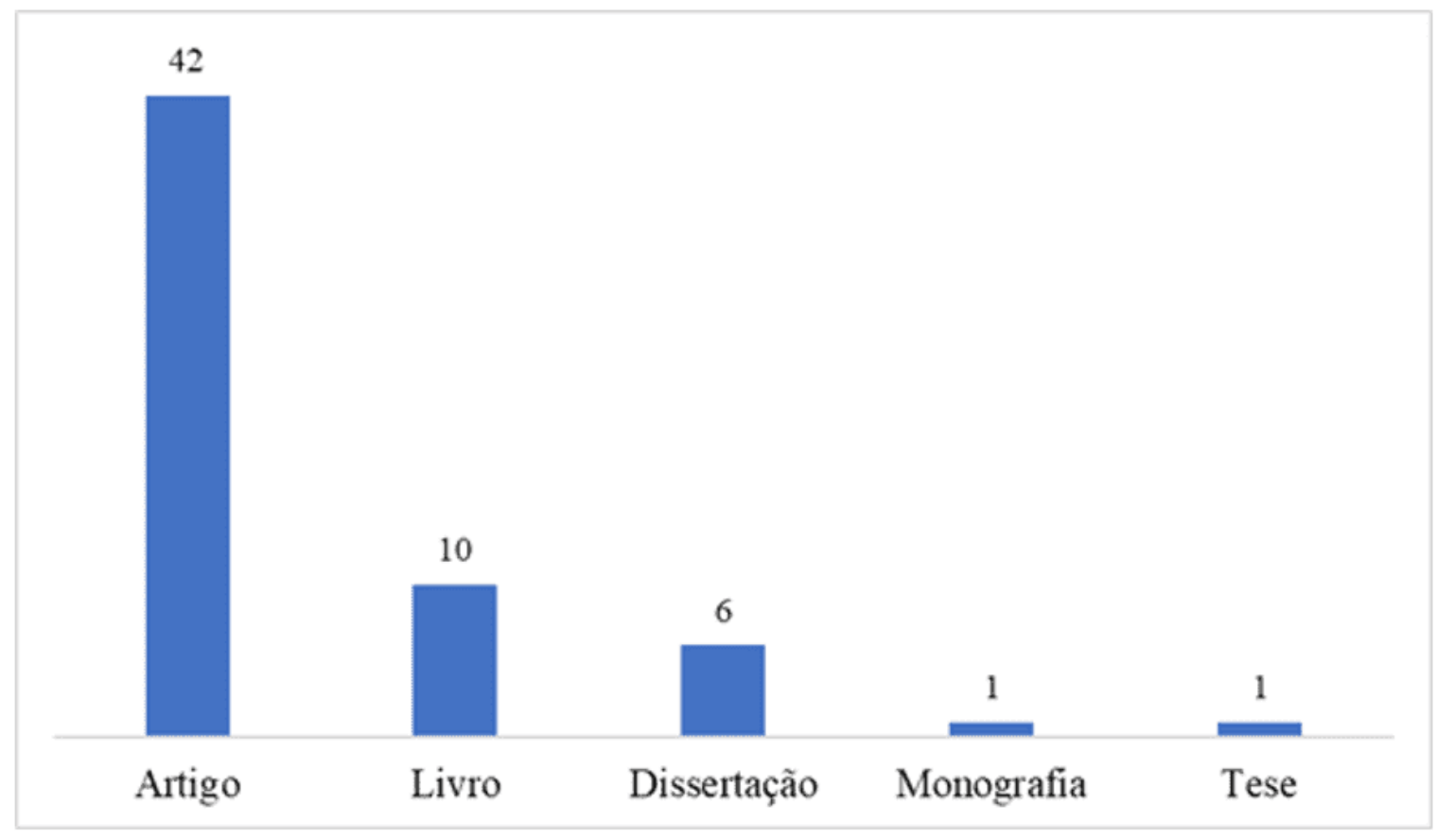

Source : Élaboration propre (2021).

C. Zone de publication

La recherche universitaire au Brésil sur le plancher salarial est centrée dans le domaine de l'éducation, atteignant le taux de $80 \%$ (48 publications). Sur le nombre total de publications sur salaire plancher dans le domaine de l'éducation, 32 sont des articles et 10 sont des livres. Par conséquent, la production d'articles et de livres prévaut. De plus, les domaines des sciences sociales et des sciences politiques représentent ensemble $11,7 \%$ (7 publications). 
Graphique 3 - Zones de publication

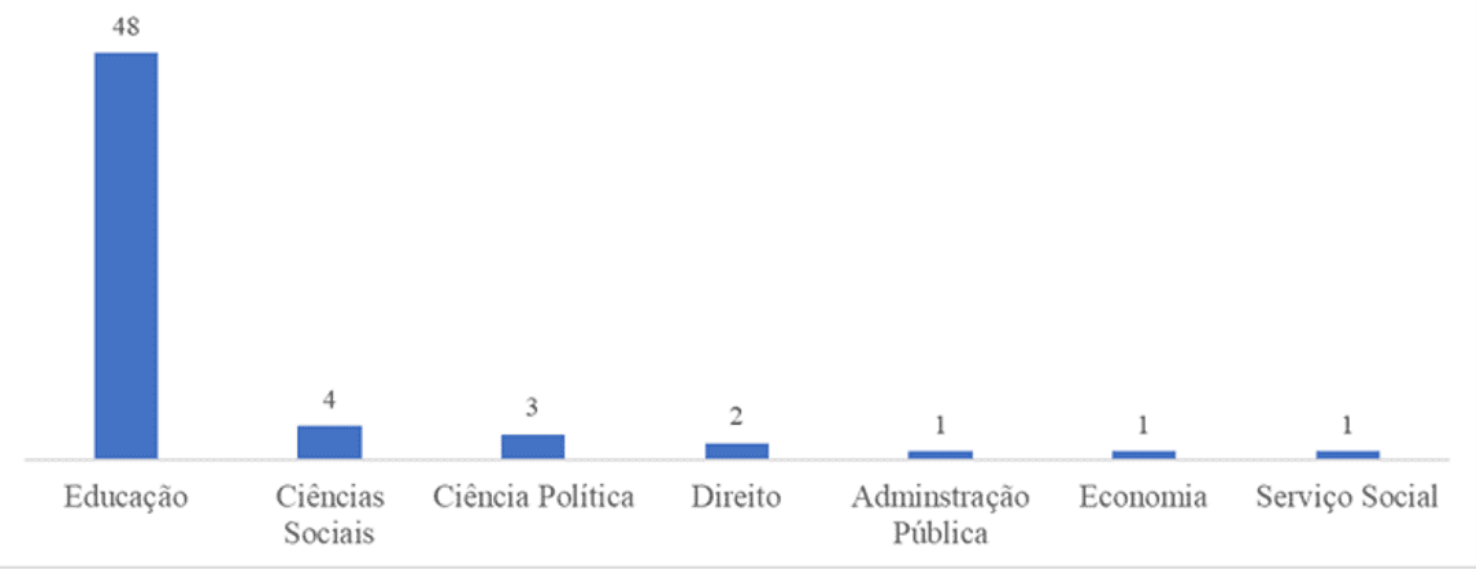

Source : Élaboration propre (2021).

Figure 4 : Zones de publication par type de publication

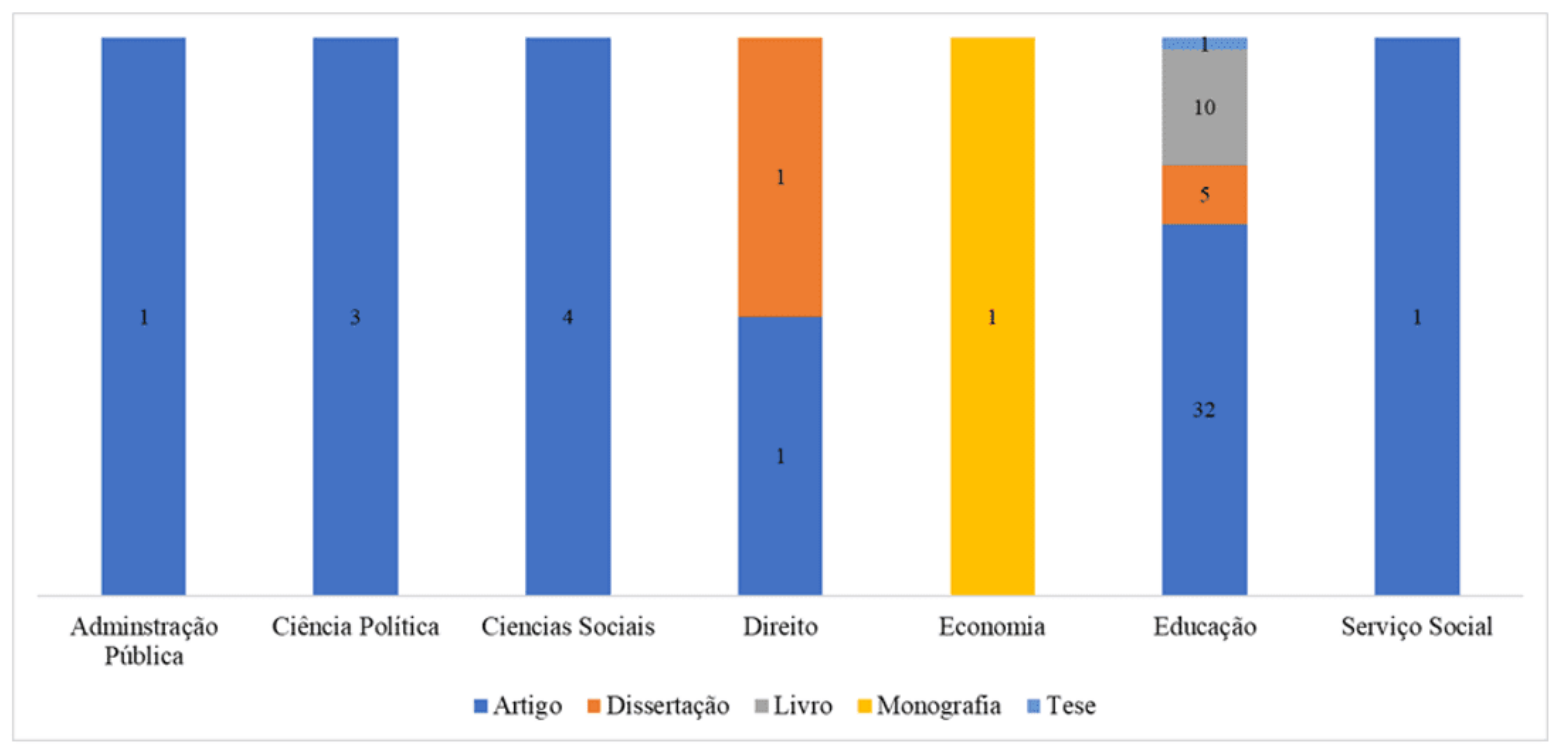

Source : Élaboration propre (2021).

D. Principales régions de publication

En élargissant l'analyse de la production académique nationale, nous avons observé si les lieux de publication d'articles, de livres, etc. étaient régionaux ou nationaux.

RC: 101662

Disponible en: https://www.nucleodoconhecimento.com.br/education-fr/remunerationdes-enseignants 
Figure 5 - Régions de publication

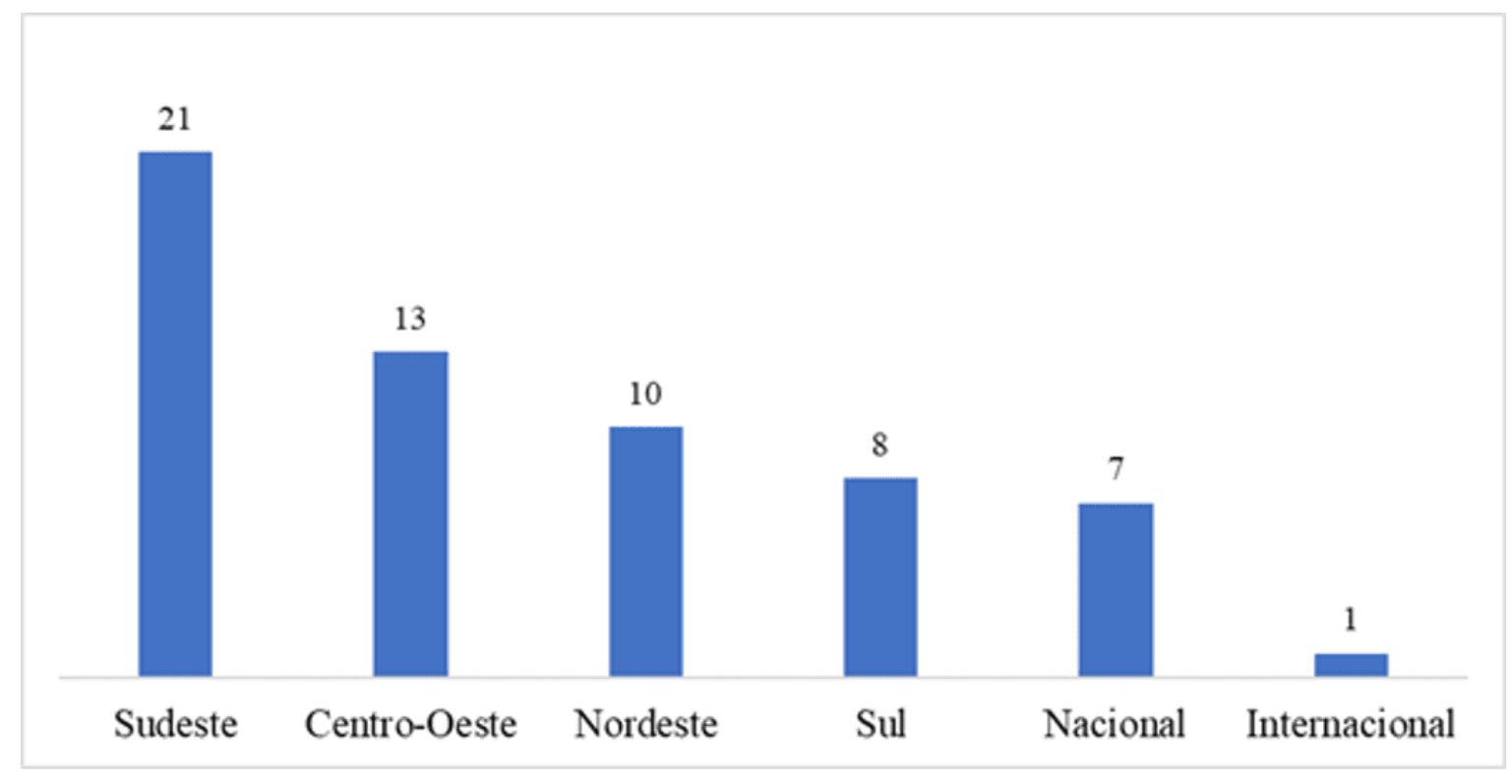

Source : Élaboration propre (2021).

La recherche bibliographique indique que la région du Sud-Est du pays est l'un des principaux lieux de publication. En plus du caractère national, les régions du Midwest, du Nord-Est et du Sud prépondérant également, par ordre décroissant.

E. Principaux lieux d'étude des publications 
Figure 6 - Régions d'étude des publications

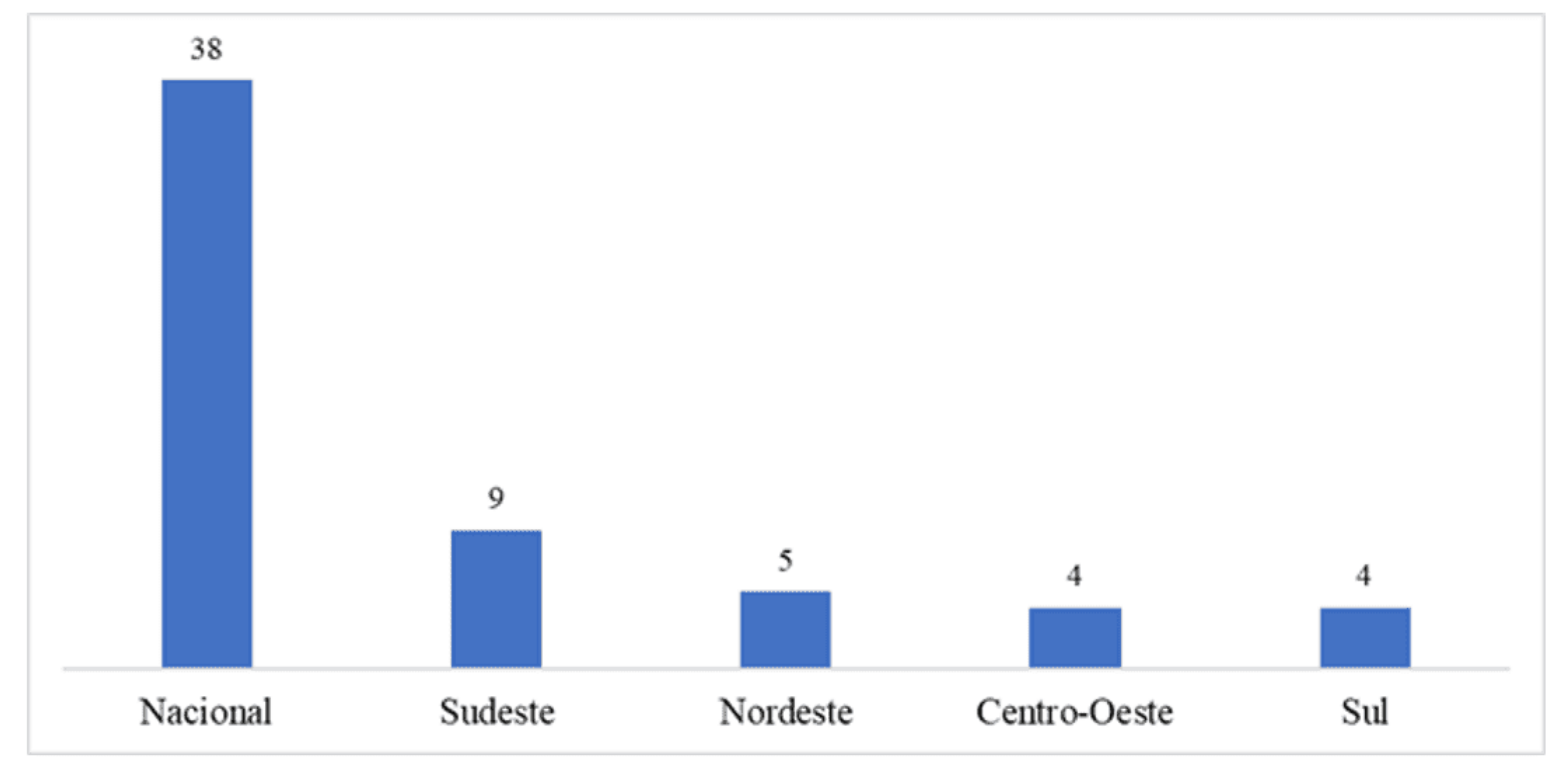

Source : Élaboration propre (2021).

Plus précisément, en analysant les titres et les résumés des publications, nous avons vérifié la tendance des études sur la loi PSPN au niveau national (63,3\%). Les analyses régionales représentent environ $36,7 \%$ de toutes les publications. La région du Sud-Est continue d'être au centre de nombreuses études et, comme nous l'avons identifié, les États de São Paulo et du Minas Gerais se distinguent. Ensuite, la région du Nord-Est émerge, représentant $8 \%$ dans l'ensemble total des publications, et il convient de mentionner les États de Bahia, Rio Grande do Norte et Piauí comme centre d'analyse des études régionales sur le plancher salarial. 
Graphique 7 - Etude de publication UF

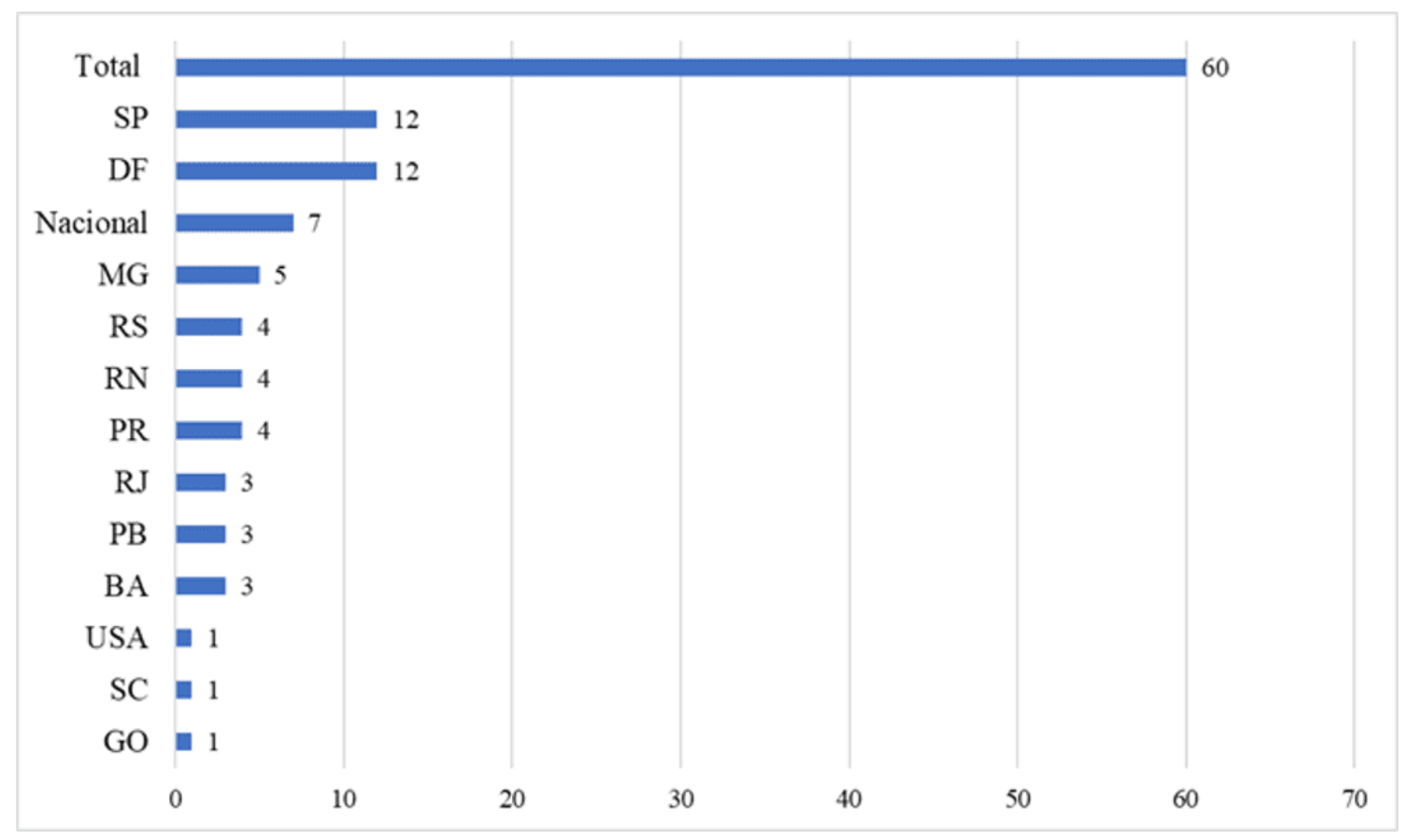

Source : Élaboration propre (2021).

F. Auteurs principaux

À partir de l'identification d'articles, de monographies, de mémoires, de thèses et de livres, il a été possible de soulever les noms des auteurs qui les ont écrits. II convient de mentionner que lorsque l'œuvre était co-auteure, comme dans certains livres spécifiques, les noms de tous étaient énumérés.

Les auteurs ont ensuite été répertoriés et regroupés pour obtenir le nombre de fois qu'un auteur donné a publié. Pour ce faire, nous avons créé une liste avec ces auteurs et développé un nuage de mots pour visualiser les plus importants. Le nuage de mots souligne, de manière directement proportionnelle, la fréquence d'apparition des mots. Ainsi, nous pouvons observer que les dix auteurs avec le plus grand nombre de publications sont: Maria Dilnéia Espíndola Fernandes, Andréa Barbosa Gouveia, Márcia Aparecida Jacomini, Claudio Pinto Nunes, Eliara Cristina Nogueira da Silva Teixeira, Ana Paula Santiago do Nascimento, Andreza Barbosa, Áurea de 
Carvalho Costa et Dalila Andrade Oliveira. Le classement des auteurs aide à la délimitation des auteurs et à la bibliographie sur le plancher salarial. Nous pouvons voir que la paternité est principalement composée de femmes.

Figure 1 - Auteurs principaux

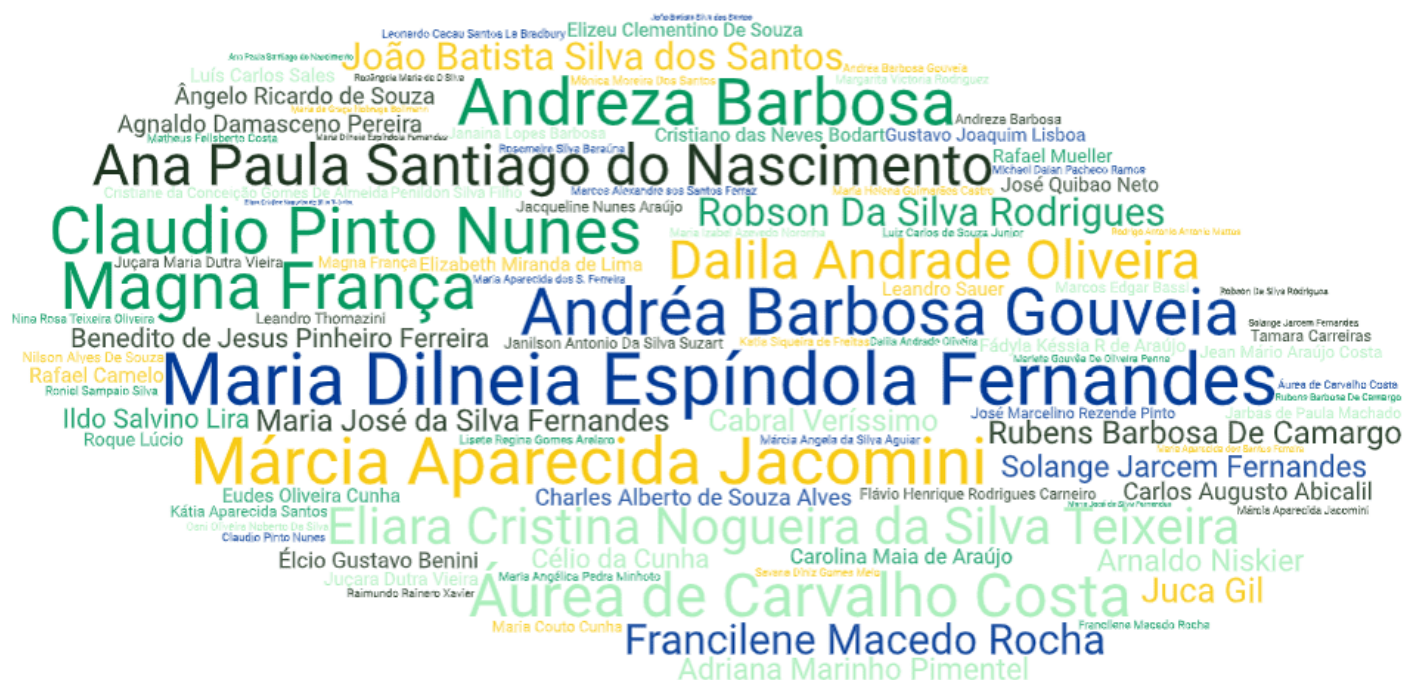

Source : Élaboration propre (2021).

\section{LE PLANCHER SALARIAL PROFESSIONNEL NATIONAL ET L'APPRÉCIATION DES ENSEIGNANTS: CE QUE DISENT LES UNIVERSITAIRES}

La revue de la littérature a un caractère qualitatif et exploratoire et a été produite pour soutenir les études doctorales de ce chercheur sur la mise en œuvre de la loi 11.738/2008, qui a créé le Plancher salarial professionnel national - PSPN. Pour contextualiser la recherche, il est important que nous sachions comment la question liée à la rémunération des enseignants a été abordée dans les productions académiques. C'est une recherche qui avait initialement pour seul but de contextualiser, de situer les auteurs sur la production et les positions académiques, mais qui a permis de comparer la production des discours en politique éducative et la vérification de leur application pratique dans le monde académique. Pour ce travail, 
nous avons sélectionné les dix articles d'auteurs qui ont produit le plus sur le terrain, la rémunération des enseignants et le respect de la loi PSPN, entre 2008 et 2021. Ces articles traitent de la rémunération des enseignants et de leurs relations avec les fonds de financement de l'éducation de base.

Après tout, que disent les universitaires de la rémunération des enseignants, étant donné qu'il y a eu près de deux siècles de luttes pour le plancher salarial professionnel national?

Le 15 octobre 1827, Dom Pedro I a établi, à l'article 3 de la loi sur les écoles de premières lettres, le premier plancher salarial pour l'enseignant, mais cela n'a jamais été appliqué. Depuis la Constitution de 1988, les discussions syndicales et universitaires ont pris des contours de plus en plus prononcés. Peu d'experts en éducation ont dissocié la lutte pour la démocratisation de la lutte pour l'école publique gratuite, avec des enseignants ayant une formation initiale solide et un salaire décent. Le 16 juillet 2008, le président de l'époque, Lula, a sanctionné la loi 11 738/2008, qui établissait l'étage de salaire professionnel national pour les professionnels de l'éducation publique de base, établissant une valeur à ajuster obligatoirement chaque année et «en dessous de laquelle l'Union, les États, le District fédéral et les municipalités ne seront pas en mesure de fixer le salaire initial des carrières de l'enseignement public de l'éducation de base, pour un trajet d'un maximum de 40 (quarante) heures par semaine »(BRASIL, 2008). Bien que le plancher soit établi par la loi depuis 2008, plus de 10 ans après sa sanction dans plus de la moitié des municipalités, plus de la moitié des municipalités brésiliennes violent la loi sur les sols, sans aucune sanction.

Il y a eu deux siècles de luttes des enseignants pour la création du plancher salarial pour le magistère, mais il y avait encore des tentatives de certains gouverneurs pour empêcher leur sanction, qui ont heureusement été frustrées.

Lors d'un travail de consultation effectué pour l'Unesco/MEC, nous avons constaté que sur 5570 municipalités, seulement 30\% se conforment à la loi 11.738/2008 dans 
son intégralité, c'est-à-dire payaient l'étage initial, créaient de nouveaux plans de carrière, assurent $30 \%$ de la charge de travail pour les études et la planification, en plus d'assurer une formation initiale solide et une entrée par appel d'offres public. Par conséquent, nous avons décidé d'étudier la mise en œuvre du plancher salarial, et dans le cas de la thèse de doctorat[4] que nous développons, en particulier sur l'État de Bahia, qui est le 4ème plus grand État de la Fédération et le plus grand du Nord-Est brésilien. Dans un premier temps, pour étayer les études de la thèse citée sur la mise en œuvre du plancher dans les 417 municipalités de Bahia, nous avons cherché une base de recherche bibliographique qui a atteint des dizaines d'études. Pour cet article, nous présentons les dix auteurs qui ont produit le plus sur le sujet au cours de cette période. Dans ce contexte, l'objectif de cet article est de présenter le développement de cette ligne de recherche, que nous considérons comme pertinente. Nous soulignons ses questions centrales, ses avancées et ses limites, bref, l'état de l'art de la littérature sur PSPN.

Malgré le dilemme qu'impliquent les influences de la politique sur les politiques publiques pour les enseignants et pour une éducation de qualité, dans un pays de " démocratie restreinte ", comme l'a souligné Florestan Fernandes (1987, p. 160), nous insistons sur la recherche de questions qui nous permettent d'identifier la place occupée par l'appréciation de l'enseignant et de l'éducation dans l'univers académique. Pour répondre à la question sur l'intérêt des études menées sur la mise en œuvre du Plancher Salarial Professionnel National - PSPN et si celles-ci indiquent les répercussions du plancher salarial sur la valorisation du magistère, dans la période 2008-2021, nous avons recherché des articles, des monographies, des mémoires et des thèses dans le domaine des sciences sociales ou du domaine de l'éducation.

Au cours des quarante dernières années, des auteurs plus importants, bien que peu nombreux, se sont engagés dans l'analyse des statuts, des programmes et des pratiques des gestionnaires et ont cherché à comprendre l'influence des partis politiques pour expliquer les variations dans les résultats des actions gouvernementales dans le domaine de l'éducation. Dans cette période, sans aucun RC: 101662

Disponible en: https://www.nucleodoconhecimento.com.br/education-fr/remunerationdes-enseignants 
doute, les études sur les pratiques et les politiques éducatives viennent inclure l'enseignant, auparavant presque invisible. La preuve en est les chapitres de la Constitution fédérale et des Constitutions des États qui soulignent l'appréciation de l'enseignant; la loi sur les lignes directrices et les bases de l'éducation nationale; la définition des Lignes directrices nationales pour la formation des enseignants; les Plans Nationaux d'Éducation du Brésil - PNE (2011-2020) et PNE (2014-2024) et la Loi 11.738/2008, qui a créé le plancher salarial, documents pour lesquels la qualité de l'éducation et l'appréciation des enseignants constituent de réels défis. Dans le nouveau Plan national d'éducation du Brésil - PNE (2014-2024), sur les 20 objectifs décrits, 4 concernent l'enseignant, bien que les plans nationaux d'éducation aient été mis de côté par les dirigeants des sphères fédérale, étatique et municipale, et soient transformés en simples protocoles d'intentions.

Ensuite, nous passons à la recherche des universitaires sur le PSPN, sur sa mise en œuvre, sa rémunération et l'appréciation du magistère.

L'Unesco a publié, au cours de la dernière décennie, deux ouvrages à couper le souffle: Professores do Brasil: impasses e desafios (2009), de Bernardete Angelina Gatti et Elba Siqueira de Sá Barretto, et Políticas Docentes - um estado da arte (2011), de Bernardete Angelina Gatti, Elba Siqueira de Sá Barretto et Marli Eliza Dalmazo Afonso de André. Ces études apportent une radiographie des travailleurs de l'éducation. Professores do Brasil: impasses e desafios (2009) montre la dimension du défi qu'est la valorisation des enseignants et souligne que " les enseignants sont parmi les groupes professionnels les plus nombreux ". Et il y a encore "les défis de surmonter les commodités politiques et d'adopter des stratégies articulées entre les différents organes de formatage ». En conclusion, les auteurs utilisent le mot " intrigue », qui est un défi théorique et qui s'insinue dans le cadre de notre travail. En 1992, nous avons publié la monographie intitulée " $A$ trama da ignorância » (LIMA, 1996), dans laquelle nous analysions les discours des autorités brésiliennes sur l'enseignant, dans l'Empire et dans la République, en les confrontant aux politiques efficaces de valorisation de l'enseignant. En 2011, les chercheurs Bernardete Angelina Gatti, Elba Siqueira de Sá Barretto et Marli Eliza 
Dalmazo Afonso de André ont publié l'étude Políticas Docentes no Brasil - un état de l'art, publié par l'UNESCO et d'une grande portée. Dans cette étude, les auteurs cartographient et analysent les politiques d'enseignement appliquées dans les différentes sphères fédératives - Union, États et Municipalités - et placent au centre de la discussion la formation initiale et continue de l'enseignant, la carrière de l'enseignement et l'évaluation des enseignants. Dans les conclusions, ils parlent de la diversité des propositions pour l'appréciation des enseignants, mais ne se réfèrent pas à la mise en œuvre complète ou non de la loi $11.738 / 2008$, qui a créé le Plancher national des salaires professionnels - PSPN -. Et ils avertissent:

Se não houver aderência das propostas às políticas próprias dos estados e dos municípios, no caso das políticas federais, e destes últimos, no caso das políticas estaduais que a eles se estendem, ficam comprometidas a possibilidade de desenvolvimento profissional dos docentes e a sustentação das conquistas adquiridas (GATTI; BARRETO; ANDRÉ, 2011, p. 266).

Le débat académique sur la carrière et la rémunération des enseignants a fait l'objet de recherches des auteurs Rubens Barbosa de Camargo, Andréa Barbosa Gouveia, Juca Gil et Maria Angélica Pedra Minhoto (2009). Dans cette recherche, les auteurs analysent les relations entre les résultats du Fundef sur la base d'indicateurs économiques et la rémunération des enseignants, en ayant comme paramètres les références juridiques sur le plancher salarial professionnel national. Pour cela, les auteurs ont utilisé " indicateurs économiques nationaux, salaires des enseignants dans les réseaux de l'État et valeurs des années-étudiants de Fundef ». Et ils ont conclu que les variations salariales sont liées à chacun des États, présentant une inflexion positive vers la recomposition des salaires, au-dessus de l'inflation. Ils soulignent la " nécessité d'études complémentaires pour comprendre la dynamique de la rémunération ». Sur la nécessité d'études supplémentaires pour soutenir la fixation du plancher salarial, il convient de se demander si les experts ont oublié que les maîtres ont attendu deux siècles pour conquérir le plancher salarial établi par la loi, pour l'ensemble du pays. 
Pour les auteurs, "la rémunération des enseignants, en plus de devoir être mieux 'déchiffrée', pour apporter un soutien adéquat à la conception d'un PSPN, car elle est déterminée par des facteurs (externes et internes) impliquant des intérêts différents " (CAMARGO et al., 2009). Tout cela " pour exposer sa plus grande dimension explicative, la révélant comme expression concrète d'un rapport de forces en conflit de projets de société, d'école, d'homme, de valorisation de l'enseignement : bref, comme lutte politique " (CAMARGO et al., 2009, p. 360). Quels sont les intérêts qui mobilisent la non-application de la loi instituant le plancher salarial ? Les disputes exprimées ici, dont nous parle Florestan Fernandes, sont-elles entre des sceptiques de droite ou de gauche, qui ne sont d'aucune aide, et qui sabotent presque toujours l'éducation au Brésil ?

Dans un article de 2010, Santiago do Nascimento, Amorim et Camargo (2010) ont analysé la rémunération des enseignants du réseau de l'État de São Paulo au début de leur carrière et ont souligné sa composition sur la base du document $d u$ Département d'État de l'Éducation intitulé Sistema de Informações Educacionais Boletim de Acompanhamento de Pessoal (novembre 2010). Grâce à cela, ils ont pu vérifier l'évolution des réajustements des paiements reçus par les enseignants, dans la période entre 1996 et 2010.

Santiago do Nascimento, Amorim et Camargo (2010) ont constaté que «la rémunération était composée de différents bonus qui représentaient plus de $35 \%$ du total ». Et que, par rapport au salaire minimum, la rémunération a subi un aplatissement, passant de 3,6 en 1996 à 2,4 en 2010. Les auteurs ont l'intention de contribuer aux discussions sur la rémunération des enseignants au Brésil en analysant les périodes par la direction, les gouverneurs et / ou les secrétaires de l'éducation. Dans la gestion du gouverneur Mário Covas, la rémunération des professionnels de PEB I est passée de 360,94 R\$ en 1996 à 710,00 R\$ en 2000 et 2001, en valeur nominale. En comparant ces valeurs, nous avons remarqué que la rémunération a augmenté de $96,71 \%$ en cinq ans. 
Sous Alckmin, la rémunération des professionnels de PEB I est passée de 710,00 $\mathrm{R} \$$ en 2001 à 1 144,39 R\$ en 2005 (NASCIMENTO; AMORIM; CAMARGO, 2010). L'augmentation a été de $61,18 \%$ en quatre ans. L'échéance est passée de $R \$$ 610.00 à $R \$ 726.19$. Au cours de cette période, le pourcentage le plus élevé de primes a été versé, les primes atteignant $36,5 \%$ de la rémunération des enseignants.

Pendant la période du gouvernement de José Serra, les paiements initiaux des enseignants de PEB I sont passés de 1 144,39 R $\$$ en 2008 à 1 198,16 R $\$$ en 2010, en valeur nominale. En analysant les salaires ( $R \$ 726,19$ et $R \$ 981,88)$, nous avons remarqué une augmentation de $35,21 \%$. La représentativité des primes dans le gouvernement Serra est passée de $36,5 \%$ en 2008 à 18,1\% en 2010. Cela signifie que ce gouvernement avait une politique d'intégration des primes dans le salaire, mais avec une possible perte réelle de pouvoir d'achat, selon la relation avec le salaire minimum. La représentativité des primes dans le gouvernement Serra est passée de $35,5 \%$ en 2008 à $17,4 \%$ en 2010.

Ainsi, les auteurs concluent que tout au long de la période étudiée, lorsqu'on prend comme indicateur le salaire minimum (ou le salaire nécessaire de Dieese), une forte oscillation a été perçue. Cependant, lorsque nous considérons les années 1996 et 2010, nous avons remarqué qu'il y avait moins de salaire minimum, que ce soit en salaire ou en rémunération initiale, ce qui indique un aplatissement des revenus des enseignants de São Paulo.

En ce qui concerne la politique d'appréciation des enseignants, les tensions sont élevées. Dans un article publié par Maria Dilnéia Espíndola Fernandes et Margarita Victoria, intitulé «O processo de elaboração da Lei ํo 11.738/2008, Lei do Piso Salarial Profissional Nacional para carreira e remuneração docente: trajetória, disputas e tensões ", les auteurs sauvent la trajectoire récente de la construction de la loi nationale sur les salaires professionnels (PSPN) pour la rémunération des carrières et des enseignants au Brésil, dans le cadre des réformes de l'État brésilien. Ils ont examiné la loi fédérale, les documents fédéraux et syndicaux et la littérature sur le sujet. Ils ont souligné l'une des confrontations politiques les plus importantes 
dans la construction des relations fédératives brésiliennes à ce moment historique, qui était une action directe d'inconstitutionnalité (Adin) contre la loi n ${ }^{\circ} 11$ 738/2008, déposée par les gouverneurs des États du Mato Grosso do Sul, du Paraná, de Santa Catarina, du Rio Grande do Sul et du Ceará. Dans l'action directe en inconstitutionnalité, les gouverneurs ont remis en question le plancher salarial national des enseignants des écoles élémentaires publiques, établi par la loi fédérale 11.738/2008, en discutant de la portée de l'expression «plancher » (art. 2, caput et $\S 1^{\circ}$ ); la limitation du montant versé à titre de salaire de base initial de la carrière ou la prolongation du salaire global; et la définition de la charge de travail. Et ils ont allégué la violation de la loi de réserve d'initiative du chef de l'exécutif pour disposer du régime juridique du fonctionnaire (art. 61, $\S 1$, ii, $\mathrm{c}$ de la constitution); contrairement au pacte fédératif (art. 60, § 4 et article de la constitution) ; nonrespect de la règle de proportionnalité : le jour ouvrable et le salaire fixés par la loi. Les auteurs ont compris "que le PSPN est implanté en termes de conception ". Avec le Tribunal fédéral suprême, la plus haute instance juridique du pays, ayant approuvé la loi $n{ }^{\circ} 11$ 738/2008 (BRASIL, 2008a), le litige juridique a été résolu, donnant un gain aux enseignants, leur assurant des plans de carrière pour l'enseignement public, un salaire plancher professionnel et une allocation de $30 \%$ de la charge de travail pour les études et la planification, en plus d'entrer dans la carrière exclusivement par concours public de tests et de titres. Le 17 décembre 2008, lors du vote du ministre Joaquim Barbosa, il était écrit « qu'il n'est pas crédible de supposer que les États fédérés s'opposent à la réduction des inégalités régionales, à l'amélioration des conditions dans lesquelles les services publics d'éducation sont fournis ". La mise en œuvre du plancher dans les différentes unités infranationales a montré à quel point il est difficile de surmonter les localismes de la tradition culturelle brésilienne dans la construction historique du fédéralisme. Pour eux, la viabilité matérielle du PSPN dépendra également de beaucoup d'efforts de la part des enseignants de l'éducation de base, ainsi que d'éventuels alignements dans les relations politiques autour du modèle de fédération en vigueur. Le scénario national présente des situations très différentes en ce qui concerne la question salariale des professionnels de l'éducation de base. Ce qui est commun entre les 
unités fédérées sur les salaires des enseignants, c'est le faible salaire de l'enseignant.

La spécialiste Dalila Andrade (2013) a publié l'étude intitulée " As políticas de formação e a crise da profissionalização docente: por onde passa a valorização », dans laquelle elle analyse la formation de l'enseignant et le rapport à la professionnalisation. Dans l'article, l'auteur cherche à comprendre quels sont les facteurs qui déterminent l'amélioration de l'éducation. Elle critique « certaines approches qui placent l'enseignant comme le principal responsable de la tâche éducative et, par conséquent, de ses résultats, déplaçant l'orientation des structures et des relations sociales qui impliquent le contexte scolaire »(ANDRADE, 2013, p. $51)$.

Pour Andrade (2013, p. 51), « l'institution de la loi nationale sur les salaires professionnels et la création de PARFOR sont quelques-unes des politiques orientées vers une plus grande appréciation des enseignants ». Dans cette recherche, l'auteur « cherche à discuter de certaines corrélations entre la formation initiale et la formation continue avec rémunération, dans le but de mettre en évidence leurs relations pour une plus grande appréciation des enseignants » (ANDRADE, 2013, p. 51). Et il conclut que les politiques éducatives actuelles visent à répondre aux demandes d'une plus grande appréciation des enseignants. Après la LDB 9394/96, le niveau de formation des enseignants a considérablement augmenté. Néanmoins, la loi a été modifiée permettant à l'enseignant ayant fait des études secondaires de devenir, dans certaines entités fédérales, une règle et non une exception. En cela, l'auteur observe un recul dans le plan juridique. Elle conclut, avec une certaine appréhension, en se montrant préoccupée par un écart par rapport aux piliers des politiques: la carrière; les conditions de travail et la rémunération, avec un éventuel passage « à un modèle de responsabilité des enseignants pour leur formation, en particulier la formation continue, comme forme de croissance individuelle et professionnelle » (ANDRADE, 2013, p. 69). « II y a un sentiment général que la profession enseignante subit un processus de dévaluation 》 (ANDRADE, 2013, p. 51). L'analyse des données de la recherche a montré que la 
corrélation entre l'enseignement initial supérieur (titrage) et la rémunération est sûre. Contrairement à la formation continue, qui ne présente pas de corrélation sûre. II s'agit de repenser les politiques de formation continue. L'auteur souligne que faire face à la crise de la carrière enseignante nécessite des mesures efficaces pour renforcer la carrière, tant dans les formes d'entrée que dans la permanence de l'enseignant, dans la carrière, ce qui suppose des conditions de travail et de rémunération.

Nous voyons que les politiques éducatives actuelles devraient chercher une articulation entre les politiques de formation et les conditions de professionnalisation, en donnant plus de poids à une formation initiale solide dans la définition et la certification de la profession. L'ancien ministre de l'Education Rossieli Soares, du gouvernement du président Michel Temer, a présenté, au Sénat fédéral, le 15 mai 2018, un document intitulé «Panorama da Educação Brasileira ». Dans le document, la MEC a déclaré 57 millions d'inscriptions dans le réseau public, mais a dénoncé la très faible qualité de l'éducation publique, soulignant parmi les causes le pourcentage élevé d'enseignants ayant une formation inadéquate, allant de $30 \%$ à $40 \%$. Et la moitié des professeurs de mathématiques n'avaient aucune formation sur le terrain. L'ancien ministre a également indiqué que $80 \%$ des enseignants de l'enseignement de base sont formés dans des institutions privées. Dans les cours de pédagogie, sur les 690780 inscriptions, $80 \%$ d'entre elles se font dans le réseau privé. Selon Rossieli, les universités fédérales doivent comprendre que la formation des enseignants est une stratégie nationale de développement.

La chercheuse Maria Dilnéia Espíndola Fernandes (2013), dans l'article « La valorisation des professionnels de l'éducation de base dans le contexte des relations fédératives brésiliennes », analyse, à partir d'instruments juridiques et juridiques, la valorisation des professionnels de l'éducation de base à travers la mise en œuvre de Fundeb et du Plancher salarial professionnel national (PSPN) dans le contexte des relations fédératives brésiliennes. Après avoir mené une recherche documentaire et examiné la littérature sur le sujet, a. L'auteur a constaté que dans les relations fédératives brésiliennes, les politiques éducatives pouvaient contribuer à la 
collaboration fédérative concernant la réduction des inégalités sociales. L'auteur a conclu que "l'exercice du pouvoir local est encore souvent un obstacle à la coordination fédérative en matière de politique éducative " et que cela « déterminerait le fort pouvoir d'incitation de l'Union face, entre autres, à la mise en œuvre du PSPN » (FERNANDES, 2013).

En 2016, Andreza Barbosa et Maria José da Silva Fernandes, dans l'article intitulé « A valorização dos profissionais da educação básica no contexto das relações federativo brasileiro ", publié dans le magazine Retratos da Escola, ont discuté de la situation actuelle des salaires et des heures de travail des enseignants dans le réseau public de l'État de São Paulo pour vérifier l'adéquation à la loi $n{ }^{\circ} 11738$, 2008, qui a créé le plancher salarial du magistère. Les auteurs ont identifié plusieurs preuves révélant « que le gouvernement de l'État contourne systématiquement la législation fédérale, conformément aux heures de travail des enseignants à la loi sur les planchers, et paie, cette année-là, une rémunération inférieure à celle légalement établie, ce qui augmente la dévaluation des enseignants " (BARBOSA; FERNANDES, 2016, p. . 243).

Le travail de recherche intitulé «Financiamento da Educação e Luta Sindical: conflitos em uma grande rede de ensino », par Andréa Barbosa Gouveia et Marcos Alexandre dos Santos Ferraz, portant sur l'impact de la loi nationale sur le plancher salarial professionnel sur les conflits des enseignants à Curitiba avec le responsable du réseau local de l'éducation, a analysé les agendas de réclamations formulées par le syndicat des enseignants entre 2008 et 2012. Les auteurs ont concentré leur attention sur les éléments liés au revêtement de sol et à la rémunération et à la mise en œuvre du $1 / 3$ d'heure d'activité. II a été possible d'observer différentes manières de vocaliser les exigences de parole et différents impacts concrets sur la rémunération. À certains moments, provoquant l'aplatissement de la carrière et, dans d'autres, l'aplatissement du sol lui-même. Quant à l'activité horaire, elle cesse d'être une revendication d'organisation du travail et assume la caractéristique d'un droit équitable. Les auteurs ont conclu que la façon dont les ordres du jour des réclamations ont été construits a changé après le réajustement du plancher - et que,

RC: 101662

Disponible en: https://www.nucleodoconhecimento.com.br/education-fr/remunerationdes-enseignants 
à partir de 2012, "après avoir changé la façon d'expliquer la réclamation du plancher, l'indice d'ajustement commence à se concentrer sur l'ensemble du tableau des salaires, sans distinction. Ainsi, l'effet d'aplatissement de la carrière est contenu "- et que la mise en œuvre d'une activité de $1 / 3$ heure devient un thème important, frappant dans les revendications. Dans les ordres du jour antérieurs à 2011, le temps d'activité était limité à être un programme de contrôle du respect de la législation municipale déjà en vigueur. Jusqu'en 2011, les demandes d'ajustement salarial suivaient la logique de la somme d'un ensemble d'indices de pourcentage - indice d'inflation de la période, indice de gain réel, indice de perte historique. Et jusqu'en 2011, l'ajustement du niveau 1 de la carrière (c'est-à-dire le plancher) se comportait toujours différemment des autres niveaux. C'est-à-dire que le plancher pour l'enseignant diplômé est plus élevé que ce qui se passe dans les autres points du tableau des salaires (exception faite à l'année 2010). Cela signifie qu'il y a eu une appréciation du sol, mais avec un aplatissement de la carrière. L'aspect intéressant est que dans la somme des années 2009, 2010 et 2011, le niveau 1 de la carrière a un ajustement plus élevé que l'ajustement du plancher national dans la même période. Depuis 2012, ce concept de somme de plusieurs indices est remplacé par une valeur nominale de plancher unique. L'effet de ces deux stratégies distinctes est différent lorsque l'on observe le résultat final des négociations. Les auteurs concluent également que l'ordre du jour prend la dimension de revendiquer un droit prescrit par la loi et concluent en déclarant: "le cas spécifique du Réseau municipal de Curitiba et du SISMMAC montre que les enseignants sont en constante évaluation de ses effets, ainsi que de réévaluer leurs propres tactiques et stratégies de lutte pour pouvoir jouir d'un droit que la société brésilienne leur a accordé comme équitable » (GOUVEIA; FERRAZ, 2016).

L'un des articles les plus intrigants a été publié en 2016 dans la revue Pro-posições, intitulé " Análise da carreira docente e valorização do magistério: condições de trabalho e desenvolvimento profissional », rédigé par deux universitaires qui figurent sur la liste des dix auteurs qui ont le plus publié sur la valorisation du magistère, Márcia Aparecida Jacomini et Marieta Gouvêa de Oliveira Penna. Les auteurs ont 
abordé plusieurs aspects liés aux conditions de travail des enseignants de l'enseignement de base au Brésil et à leur développement professionnel, en utilisant les données de la recherche «Remuneração de professores de escolas públicas de educação básica: configurações, impasses, impactos e perspectivas ", menée dans 12 États brésiliens et leurs capitales. Après avoir analysé les plans de carrière, vérifié la formation requise pour l'admission, les heures de travail, les incitations à la formation continue et à la progression de carrière, les auteurs déclarent que «malgré l'importance des enseignants pour la promotion d'une éducation de qualité, plusieurs problèmes doivent encore être résolus afin d'assurer des conditions de travail efficaces pour les enseignants » (JACOMINI; PENNA, 2016). Les auteurs concluent l'article en citant une recherche sur le statut de l'enseignement dans des pays tels que l'Argentine, le Brésil, le Pérou et l'Uruguay, par Emílio Fanfani (2007, apud JACOMINI; PENNA, 2016), dans lequel il souligne que

toda política docente deve ser integral. Isto quer dizer que deve contemplar intervenções articuladas em pelo menos três dimensões: o recrutamento e a formação inicial e permanente, as condições de trabalho (divisão do trabalho pedagógico, carreira, contexto institucional, de trabalho, etc. e o sistema de estímulo e recompensa $s$ materiais e simbólicas (salário e reconhecimento social).

Dans un autre paragraphe des dernières considérations, les auteurs susmentionnés concluent que « les recherches présentées au Brésil sur les conditions de travail de l'enseignant indiquent une dévaluation politique et sociale de l'enseignement structurant une telle pratique professionnelle ». Et ils affirment, sans entrer dans le fond, que « l'enseignant doit avoir le salaire en ligne avec la valeur et l'importance que ces professionnels ont dans la société contemporaine ». La recherche indique la dévaluation des enseignants avec la société contemporaine et que pour changer cette situation, il est nécessaire de reconnaître et de valoriser la société.

L'analyse des «Intencionalidades das políticas de valorização docente que foram efetivadas nos governos FHC e LULA, a partir das políticas de Fundos (Fundef e Fundeb) » a fait l'objet d'une étude publiée par Teixeira et Nunes (2016). Dans cet article, les auteurs examinent la question du salaire et de la carrière des enseignants 
de l'éducation de base à travers l'analyse de la place occupée par le Plancher salarial professionnel national (PSPN) dans les agendas des gouvernements FHC et Lula. Ils ont également vérifié si les politiques appliquées par ces gouvernements contribuaient à l'amélioration des salaires des enseignants brésiliens. Et ils concluent que le plancher salarial national en tant que mesure de valorisation du magistère a reçu des approches différentes dans les gouvernements FHC et Lula. Le Fundef, dans le gouvernement $\mathrm{FHC}$, bien qu'il ait provoqué une amélioration des salaires des éducateurs, n'appréciait pas « une référence nationale pour le début d'une carrière, ce qui a amené ce Fonds à ne pas atteindre son objectif de valoriser l'enseignement de l'éducation de base ». D'autre part, Fundeb, dans le gouvernement de Lula, en plus de maintenir un minimum de $60 \%$ des ressources pour le paiement des enseignants, a institué la période d'un an pour la création du plancher salarial dans une loi spécifique, qui a abouti à la loi $n{ }^{\circ} 11738 / 2008$, qui a établi la mise en œuvre du plan de position (TEIXEIRA; NUNES, 2016, p. 252).

Dans un article de Robson da Silva Rodrigues et Áurea de Carvalho Costa (2019) intitulé « Da Constituição Federal de 1988 ao Plano Nacional de Educação 20142024: ardilosas apropriações da noção de valorização do trabalho docente ", les auteurs concluent que les politiques mises en œuvre entre 1988 et 2014 ont favorisé la dévaluation du magistère. La recherche avait comme référence théorique le matérialisme historico-dialectique. Les auteurs disent qu'en observant l'activité éducative dans les pratiques quotidiennes, « ceux qui mettent en œuvre la pratique éducative contredisent les politiques éducatives qui prétendaient viser à valoriser les enseignants. Cette contradiction provient de l'application de notions de valorisation vidées de leur sens axiologique. Dans ces cas, ainsi que dans la législation de l'État, « la reconnaissance de la valeur de l'enseignant est liée à des prescriptions externes sur son travail et qui se traduisent par des récompenses sous forme de rémunération, d'avantages sociaux et de progression de carrière, liées à la culture de la performance » (RODRIGUES; CARVALHO, 2019, p. 231).

Dans une étude menée par Andréa Barbosa Gouveia et Maria Dilneia Espíndola Fernandes (2019), sur la base des données rapportées par la Liste annuelle RC: 101662

Disponible en: https://www.nucleodoconhecimento.com.br/education-fr/remunerationdes-enseignants 
d'information sociale (RAIS, 2016) sur les salaires des enseignants dans les États du Mato Grosso do Sul et du Paraná, il a été démontré que la mise en œuvre et le maintien de l'ensemble national des salaires professionnels étaient dus à l'introduction du gouvernement fédéral et à l'action syndicale qui en a résulté. L'agenda syndical de l'enseignement incorporait la défense du principe de valorisation professionnelle et construisait la nécessité d'un plancher salarial professionnel national (PSPN) comme élément structurant. La réglementation nationale de PSPN a eu lieu en 2008; cependant, en raison du pacte fédératif brésilien, le respect de la législation dépend des gouvernements locaux. Cela impliquait une large mobilisation des syndicats d'enseignants afin que la définition de la rémunération intègre la règle nationale. Pour les auteurs, "le scénario de crise institutionnelle mis en place en 2015, suivi d'un agenda économique restrictif pour les droits sociaux, a mis en péril l'ensemble des conditions durement gagnées pour la valorisation des enseignants »(GOUVEIA; FERNANDES, 2019, p. 89).

Dans une recherche menée au Réseau municipal d'éducation de Belo Horizonte RMEBH, les spécialistes Francilene Macedo Rocha et Savana Diniz Gomes ont conclu que " la loi du PSPN, contrairement à la valorisation du magistère qui recommandait, a induit une baisse de la rémunération et une restriction de l'horizon des luttes des enseignants pour la carrière unique des enseignants de RMEBH, conquise en 1996 » (ROCHA; MELO, 2019, p. 3). Pour les auteurs, avec la politique du Plancher salarial professionnel national, il y a eu un processus de précarité du travail d'enseignement dans RMEBH, de la segmentation de la carrière d'enseignant dans l'enseignement primaire et l'enseignant pour l'éducation de la petite enfance, ce qui a brisé la logique de la carrière unique des enseignants de RMEBH. Cet article présente une discussion sur les changements dans la carrière et la rémunération des enseignants du réseau municipal d'éducation de Belo Horizonte (RMEBH) à partir de 2003, avec la création du poste d'éducateur d'enfants (EI), et à partir de 2008, avec la mise en place de la loi nationale sur les salaires professionnels (PSPN). La période étudiée s'est déroulée de 2003 à 2016. Pour l'analyse, les chercheurs avaient comme référence théorique des auteurs critiques classiques, tels que Karl 
Marx et Friedrich Engels, et des contemporains, tels que Oder dos Santos, João Bernardo et Ricardo Antunes, entre autres. Ils étaient basés sur la bibliographie de la région, sur des recherches documentaires axées sur les lois nationales et municipales sur la carrière et la rémunération et sur les publications du Syndicat des travailleurs de l'éducation du Réseau public municipal de Belo Horizonte (Sind-REDE / $\mathrm{BH})$.

L'article "Remuneração docente: efeitos do Plano de Cargos, Carreira e Remuneração em contexto municipal ", par Maria Dilnéia Espíndola Fernandes, Solange Jarcem Fernandes et Viviane Gregório de Campos (2016), publié dans la revue Ensaio: Avaliação e Políticas Públicas, a été préparé à partir d'une étude de cas. Dans cette étude, les auteurs ont cherché à vérifier l'effet produit par les plans d'emploi, de carrière et de rémunération (PCCR) mis en œuvre dans la municipalité de Campo Grande, entre 1996 et 2016, en termes de rémunération salariale pour un enseignant de plus de 20 ans de carrière. L'analyse de l'évolution de la rémunération des enseignants a été faite à partir de l'examen de leurs mérites, par rapport au PCCR et au salaire minimum. Les auteurs ont constaté qu'il y avait un impact positif sur la rémunération de la profession enseignante, avec le PCCR municipal obligatoire, et que le diplôme et le temps de travail étaient importants pour la valorisation de l'enseignant. Cependant, le paiement intégral du PSPN (Plancher salarial professionnel national) est toutefois resté dans une histoire dans la municipalité, qui devrait également être garantie par le PCCR.

\section{QUELQUES CONSIDÉRATIONS}

La collecte d'articles sur la pertinence des études concernant la mise en œuvre intégrale du Plancher national des salaires professionnels - PSPN pour les professionnels de l'éducation de base corrobore, dans une certaine mesure, les résultats. En ce qui concerne la dévaluation de l'enseignant et la compréhension du sens de l'évaluation des répercussions du PSPN sur sa valorisation, les conclusions confirment les études qui considèrent les modèles institutionnels, les revendications sociales, les formes de gouvernement, les politiques des partis et les spectres 
politico-idéologiques comme des influenceurs des résultats, bien que ce ne soient pas les seules causes. Plus important que les caractéristiques formelles de l'État et des institutions sociales est la façon dont une configuration institutionnelle influence les relations politiques.

La redémocratisation du pays, la construction d'un ensemble de lois et l'observation du fonctionnement des institutions ont transformé l'étude des politiques publiques. Cela est évident lorsque nous identifions les variables institutionnelles formelles ou formelles dans l'analyse des facteurs déterminants des politiques éducatives, tels que le comportement du pouvoir judiciaire; l'observation de la structure fédérative, avec ses obstacles; les localismes par opposition à la politique de collaboration fédérative; et les groupes d'intérêt et les affrontements de positions politicoidéologiques qui sabotent les politiques éducatives. De même, la question institutionnelle sert à identifier les similitudes entre les unités d'analyse, permettant de se concentrer uniquement sur la variation d'autres caractéristiques souhaitées, telles que les partis, les élections et les conditions socio-économiques des entités fédératives.

Nous avons observé un nombre important d'États et de municipalités qui appliquent le PSPN en fonction de leurs intérêts et de leurs revendications sociales, souvent en défaut de loi. En résumé, la complexité des politiques des partis nous a imposé la nécessité de créer une variété de modèles et de théories pour analyser leurs processus et leurs résultats, comme cela a été clair dans les discussions de chaque article. De telles stratégies analytiques, dans différentes mesures, se complètent et, en même temps, corroborent le développement conjoint et global de ce domaine de recherche sur les politiques publiques de l'éducation, en particulier sur la corrélation entre une éducation de qualité et l'appréciation des enseignants.

\section{G. Principaux sujets abordés}

La recherche bibliographique comprenait l'extraction de résumés de textes et surtout les mots-clés des articles, monographies, mémoires et thèses. Lorsque les livres ont 
été analysés, les principaux sujets de l'indexation bibliographique ont été supprimés. En agglutinant tous les sujets abordés, nous générons la fréquence de chaque motclé.

Le nuage de mots suivants vous permet de classer les thèmes sur six sujets plus pertinents. II s'agit de la politique éducative, du travail d'enseignement, du salaire plancher, du salaire professionnel national, de la valorisation de l'enseignement et de la rémunération des enseignants, qui suggèrent une grande corrélation avec l'objet d'étude de ce travail.

Figure 2 : Mots clés principaux

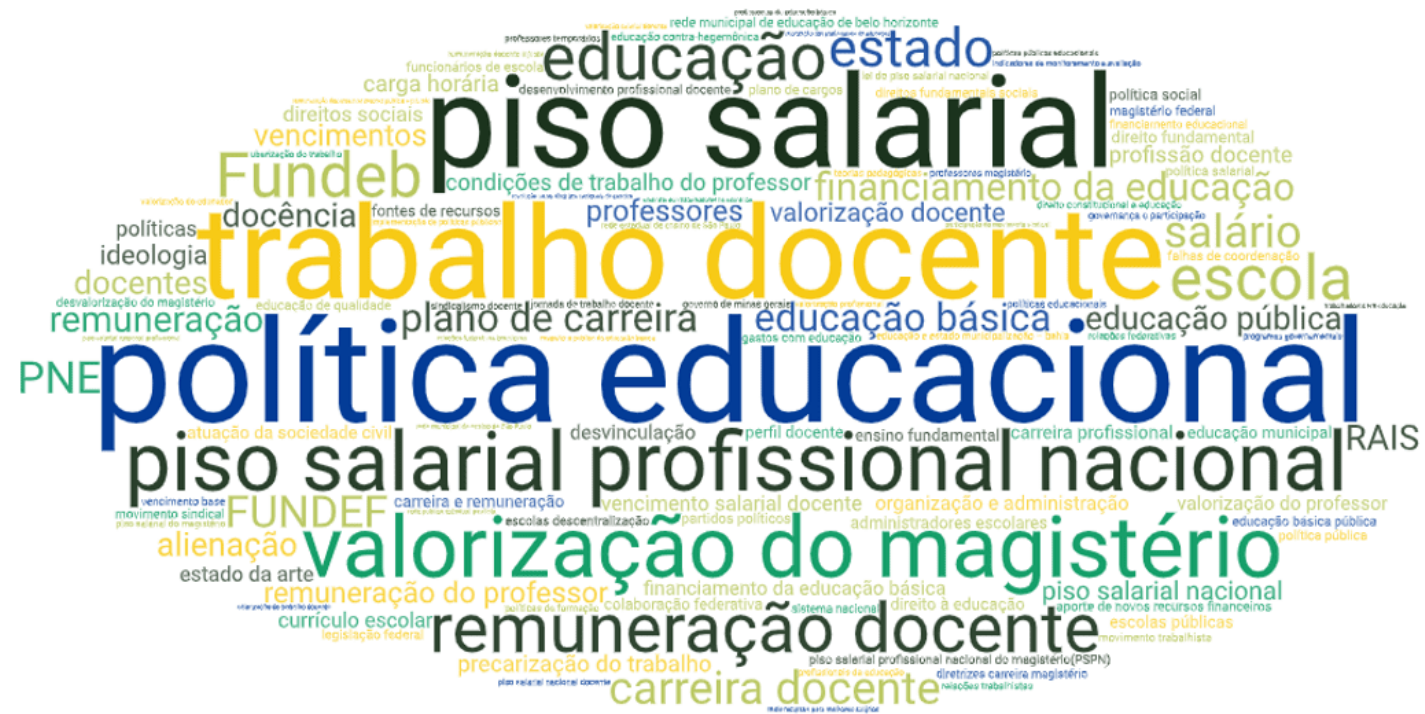

Source : Élaboration propre (2021).

Dans lesquels tant de mots du nuage pèsent qui exaltent la politique éducative, le travail d'enseignement, le plancher salarial, le plancher salarial professionnel national, l'appréciation de l'enseignement et la rémunération des enseignants, ces mots ne semblent pas maintenir la corrélation attendue dans la production académique. En effet, ils ne produisent pas d'impacts plus importants sur la production d'un nombre important de mémoires de maîtrise et de doctorat, et ces 
études sont concentrées dans des articles, ou dans des indications bibliographiques de livres.

Dans l'expérience de plus de quarante ans de militantisme dans la classe de l'enseignement primaire, intermédiaire et supérieur - en tant que dirigeant syndical dans l'État de Bahia, vice-président de la Confédération nationale des travailleurs de l'éducation (CNTE), député de l'État de 1991 à 1999, consultant législatif à la Chambre des représentants (1999 à 2003), conseiller spécial du ministre de l'Éducation (2003-2004), Conseiller spécial de la présidence de l'INEP et secrétaire adjoint à la formation professionnelle de $\mathrm{MEC}$ - ce chercheur a rencontré empiriquement la tibieza de nombreux experts en éducation et autorités éducatives, lorsqu'il s'agit de défendre des salaires décents pour l'enseignant. II connaissait également les impacts de cette conception si ancrée dans la tradition culturelle du Brésil dans la production académique peu significative sur le paiement des salaires, comme s'il s'agissait d'un thème mineur.

Au cours de ces quatre décennies de militantisme dans l'éducation, cet auteur a rencontré avec consternation deux intellectuels brésiliens de renom qui préconisait une rémunération décente de l'enseignant: le sociologue Florestan Fernandes et le philosophe Luiz Felipe Pondé. Florestan (1989, p. 58) considérait que « la déduction salariale de l'enseignant de premier degré était la plus délétère de toutes ». Et Florestan de conclure : «Peu de pays réduisent l'enseignant du premier degré à une condition aussi proche de la misère relative que le Brésil. "Pour lui, « il en va de même pour le professeur de lycée, exposé à une situation d'insécurité et de bas salaires sans équivalent. Ces diplômes d'éducation sont des piliers de l'éducation scolaire ». Et Felipe Pondé interviewé par le journaliste Thais Oyama, dans le programme Linhas Cruzadas de la Fondation Padre Anchieta (21/10/2021) dénoncé que « traiter la profession d'enseignant et l'éducation comme un thème idéalisé, comme un sacerdoce, est une stratégie pour garder l'enseignant pauvre et heureux ». Le philosophe souligne qu'« il est nécessaire de dire cela, car au Brésil, les conditions de travail de l'enseignant près auquel cas l'enseignant met fin à la vie de 
quiconque. Les salaires des enseignants sont parmi les pires au monde » (PONDÉ, 2021).

Pondé souligne que « ce traitement produit des personnes découragées ; les gens qui souffrent beaucoup pour travailler; les gens qui n'ont pas beaucoup d'horizon » (PONDÉ, 2021). Par conséquent, aussi bon que d'être dans le nuage de mots classés, cela devrait être un thème central dans les mémoires de maîtrise et de doctorat, non seulement dans le domaine de l'éducation, mais surtout dans le domaine du droit public. Après tout, ne pas se conformer à la loi sur les planchers est une transgression inassurable de la loi; devrait faire l'objet d'études de maîtrise et de doctorat en sciences sociales et politiques, car cela remet en question la politique nationale de l'éducation, la Constitution fédérale et la loi du Plan national d'éducation, consacrée par des votes au Congrès national. II est à noter que le nonrespect de la loi sur les planchers représente un démantèlement de la politique éducative, puisque 4 (quatre) des 20 objectifs de I'PNE actuelle concernent la valorisation du magistère. De plus, cela remet en question les programmes des partis, rendant un réel mauvais service à la démocratie.

Les mots magiques utilisés pour inhiber les discussions, les revendications et les études universitaires sur le salaire de l'enseignant sont: prêtrise et corporatisme. Ces stratégies ont fonctionné de manière laïque.

Par conséquent, ce résultat de ces mots expressifs, dans le nuage - dans lequel le salaire plancher, le travail d'enseignement, la politique éducative, le salaire plancher national, le plancher salarial national, la valorisation de l'enseignement, la rémunération - n'est pas articulé avec les résultats des recherches sur les nombres et les types de publication extraits des résumés des textes, les mots-clés des articles, des monographies, des mémoires et des thèses et des livres, dont les principaux sujets de l'indexation bibliographique ont été supprimés. 


\section{CONSIDÉRATIONS FINALES}

Lorsque nous arrivons à cette dernière partie de l'étude, nous élaborons des réflexions et des conclusions sur les partis politiques et l'implantation du plancher salarial professionnel national dans les municipalités - le discours et la pratique. Un chemin considérable a été emprunté à la recherche de la construction d'un cadre théorique qui permettrait non seulement d'exposer la réalité des enseignants en ce qui concerne la mise en œuvre de la loi $n^{\circ} 11738 / 2008$, connue sous le nom de Loi nationale sur les salaires professionnels - PSPN dans son intégralité, dans un court laps de temps (2008-2018). Nous cherchons également à exposer la contradiction entre les discours des politiciens qui incluent dans les statuts et les programmes des partis la défense du magistère et le contraste de leurs pratiques dans l'exercice du pouvoir. La contradiction est configurée entre les discours grandioses d'appréciation de l'enseignant et les pratiques, souvent dégradantes.

Dans aucune période historique, comme au cours des dernières décennies, le rôle de l'enseignant n'a reçu autant d'importance sur les débats sur les systèmes nationaux et les politiques éducatives et par différents acteurs, publics et privés, et les organisations nationales, internationales et multilatérales.

La recherche démontre la trajectoire des incohérences, des combats, des défaites et des victoires qui ont précédé la discussion et l'approbation de la loi n 11 738/2008. De même, il ne serait pas possible de relier uniquement les parties au respect de la loi, sans démontrer le système politique complexe des partis brésiliens, depuis ses origines.

La pertinence de ces travaux de recherche est démontrée pour évaluer les résultats des politiques d'enseignement et d'amélioration de la qualité de l'éducation, en particulier au cours de la dernière décennie. II convient de noter que le Plan national d'éducation à quatre de ses vingt objectifs directement liés à la valorisation du magistère. Et deux autres, qui ont des répercussions indirectes. 
Dans cette perspective, le choix de ce thème représentait la possibilité d'évaluer la gestion publique de l'éducation et la confrontation des discours et des pratiques des partis. En ce sens, il a été possible de vérifier l'application effective de la politique de valorisation du magistère à partir d'un outil créé par le MEC dans le but de mettre à la disposition des municipalités des instruments d'information, de suivi, d'évaluation et de suivi des politiques visant à améliorer la qualité de l'éducation: le Plan d'Actions Articulées - PAR / MEC.

Une première conclusion est que, dans laquelle tant de discussions et d'accent mis sur les politiques d'évaluation, clin d'esprit à la création d'une loi établissant le plancher salarial pour la profession enseignante, la portée de l'évaluation effective de l'enseignant est loin d'être atteinte.

L'analyse dans 417 municipalités de l'État de Bahia, réalisée à des fins de recherche visant à l'élaboration de la thèse de cet auteur[5], nous a permis de recueillir des données totales des plus grands États du nord-est du Brésil sur la mise en œuvre du salaire plancher du Magistère de Bahia, en les corrélant aux partis politiques avant les municipalités. Sur les 417 municipalités interrogées, y compris celles qui n'ont pas de parti, seules 146 (35\%) respectent pleinement la loi 11 738/2008; et sur les 271 autres, il y en a $30(7,1 \%)$ qui n'ont donné aucune information. Ainsi, on peut affirmer que 241 (57,7\%) municipalités ne se conforment pas pleinement à la Loi nationale sur les salaires professionnels.

Bien que 180 municipalités de Bahia affirment qu'elles paient la valeur du PSPN, cela ne signifie pas le plein respect de la loi, qui n'a son plein effet juridique que si elle est articulée au salaire plancher, à la définition d'au moins 33\% du travail en tant qu'activité horaire (HA), et à la garantie du plan de carrière. II est à noter l'existence d'une seule municipalité qui déclare ne pas avoir de plan de carrière, ne pas se conformer à l'HA et ne pas payer le PSPN.

Une deuxième conclusion importante, et les chiffres le montrent, est que seulement $35 \%$ des municipalités bahianaises se conforment à la loi nationale sur le niveau de 
salaire professionnel des professionnels de l'éducation - PSPN, et près de $60 \%$, plus de la moitié des municipalités bahianaises, ne se conforment pas pleinement à la loi $11.738 / 2008$, après 10 ans de sa sanction.

Peut-être que le non-paiement de la parole justifie parce que la majorité des enseignants interrogés veulent créer une carrière nationale pour l'enseignement, se plaignant d'une nouvelle loi. Le résultat de la recherche par sondage dans 38 municipalités a permis de confronter et de corroborer l'ensemble de l'enquête documentaire, ainsi que d'apporter la réalité des professionnels qui travaillent également dans des organes syndicaux, essentiels dans les luttes pour la réalisation du PSPN. Nous avons remarqué, dans les déclarations des enseignants syndiqués, même avec quelques différences, la compréhension qu'une politique de valorisation qui englobe le respect du plancher, des conditions de travail adéquates et une formation initiale et continue, serait fondamentale pour la catégorie. De même, la carrière nationale permettrait de surmonter les difficultés générées par l'autonomie décentralisée dont jouissent les entités fédérales, en raison de l'absence d'un système national articulé d'éducation.

Une autre conclusion importante de la recherche est que les différences entre les différentes parties ne sont pas significatives lorsque nous examinons les références aux enseignants dans les programmes du parti. Sur les 32 partis qui incluent l'éducation parmi les sujets abordés dans leurs programmes, seulement 12 (34,2\%) font référence aux enseignants. En cherchant ceux qui citent "valorisation, conditions de travail, salaire et formation ", il y a 10 acronymes partisans dans la distribution suivante: 2 à gauche, 3 au centre et 3 à droite. II y a peu de différences importantes entre les partis de droite, les partis du centre, les partis de centregauche et de gauche en ce qui concerne les discours dans les programmes des partis et la mise en œuvre effective de la loi 11738/2008. C'est un résultat inattendu, puisque les partis de centre-gauche et de gauche ont toujours été à l'avant-garde des luttes syndicales et parlementaires pour défendre le plancher salarial professionnel national du Magistère. 
Dans l'œuvre de Florestan Fernandes (1979), nous cherchons à comprendre la raison de cette petite différence importante entre les partis de droite, de centre et de gauche, quand il s'agit de l'appréciation de l'enseignant. En l'occurrence, en ce qui concerne l'application de politiques permanentes de valorisation du magistère et la raison de la non-application de la loi du salaire plancher du Magistère par les partis politiques, y compris ceux qui ont dans leurs programmes la valorisation de l'enseignant et en parlent comme d'un facteur décisif pour la qualité de l'éducation, ce qui explique tant de contradictions entre les discours et les pratiques des partis politiques quand il s'agit de la valorisation efficace de l'enseignant?

Pour le sociologue, fondateur de la sociologie de l'éducation brésilienne, député constitutif du Partido dos Trabalhadores Florestan Fernandes, nous avions à propos de l'enseignant un casse-tête à déchiffrer, qui consistait en la tradition d'objectivation et de brutalisation culturelle des enseignants, propre à la culture brésilienne. Après avoir cité les écrits de Marx de 1844, Fernandes ajoute que dans une comparaison de l'enseignant avec le prolétaire, il est possible d'affirmer que l'enseignant était et est toujours objectivé dans la société brésilienne. Conduit, c'est un travailleur intellectuel qui ne travaille pas seulement avec ses mains. Le défi est, comme il le dit, de chercher à comprendre cette brutalisation culturelle, qui dure depuis si longtemps, et qui est encore plus lourde lorsqu'il s'agit d'enseignants qui se consacrent à l'enseignement des enfants, ou des enseignants du primaire (FERNANDES, 1979).

Le sociologue affirme, ici et là, que les laïcs et les alphabétisés des couches sociales dominantes sont à la fois pessimistes quant à l'efficacité des institutions brésiliennes et indifférents au fonctionnement des écoles et à l'enseignement et au travail des étudiants (FERNANDES, 1979). Florestan dénonce également une profonde méfiance de l'élite vis-à-vis de l'intellectuel. Pour lui, la société brésilienne est fortement inégalitaire et très hiérarchisée. Si dans l'Empire, la démocratie des seigneurs a prévalu, dans la République la démocratie des oligarques a prévalu. La démocratie brésilienne est limitée et une culture civique est supprimée, puisque 
seule une minorité privilégiée a accès à la richesse, au pouvoir et au savoir (FERNANDES, 1989).

Ainsi, nous concluons qu'au Brésil, la tradition culturelle et le scepticisme semblent être les causes de la dévaluation des enseignants et de l'incrédulité du pouvoir transformateur de l'éducation.Selon Florestan, le scepticisme ou le dogmatisme concernant le rôle de l'école ne signifie rien dans la dynamique des transformations sociales. Pour renforcer ces paroles, l'éducateur Dermeval Saviani (1987) met en garde contre la nécessité pour les dirigeants des mouvements populaires de surmonter la vision de l'école comme un simple instrument de domination bourgeoise, qui ne fait que susciter l'intérêt de la population pour sa capacité à promouvoir l'ascension sociale. À ce stade, on peut trouver la réponse à la différence presque insignifiante entre les partis de droite, de centre et de gauche, en ce qui concerne l'application du plancher salarial des professionnels de l'éducation, qui ne se limite pas à la rémunération, mais à la carrière, aux heures de travail, à la formation et à la valorisation de l'enseignement et à l'amélioration conséquente de la qualité de l'éducation.

\section{RÉFÉRENCES}

BARBOSA, Andreza; FERNANDES, Maria José da Silva Fernandes. O Piso Salarial em São Paulo: desvalorização dos professores. Retratos da Escola, Brasília, DF, v. 10, n. 18, p. 243-257, jan./jun. 2016.

CAMARGO, Rubens Barbosa de; GOUVEIA, Andréa Barbosa; GIL, Juca; MINHOTO, Maria Angélica Pedra. Financiamento da educação e remuneração docente: um começo de conversa em tempos de piso salarial. Revista Brasileira de Política e Administração da Educação - RBPAE, v. 25, n. 2, p. 341-363, mai./ago. 2009. 
FERNANDES, Florestan. O dilema educacional brasileiro. In: PEREIRA, Luiz; FORACCHI, Marialice M. (Orgs.). Educação e sociedade: leituras de sociologia da educação. 10. ed. São Paulo: Nacional, 1979.

FERNANDES, Florestan. O dilema educacional. São Paulo: Cortez; Autores Associados, 1989.

FERNANDES, Maria Dilnéia Espíndola; FERNANDES, Solange Jarcem; CAMPOS, Viviane Gregório de. Remuneração docente: efeitos do Plano de Cargos, Carreira e Remuneração em contexto municipal. Ensaio: Avaliação e Políticas Públicas em Educação, Rio de Janeiro, v. 28, n. 106, p. 25-44, jan./mar. 2020.

FERNANDES, Maria Dilnéia Espíndola; RODRIGUEZ, Margarita Victoria. O processo de elaboração da Lei no $11.738 / 2008$, Lei do Piso Salarial Profissional Nacional para carreira e remuneração docente: trajetória, disputas e tensões. Revista HISTEDBR On-line, v. 41, p. 88-101, 2011. Disponível em: https://periodicos.sbu.unicamp.br/ojs/index.php/histedbr/article/ view/8639837. Acesso em: 26 set. 2021.

GATTI, Bernardete Angelina; BARRETTO, Elba Siqueira de Sá; ANDRÉ, Marli Eliza Dalmazo de Afonso. Políticas docentes: um estudo da arte. Brasília: Unesco, 2011.

GATTI, Elba Siqueira de Sá Barreto. Professores do Brasil: impasses e desafios. Brasília: Unesco, 2009.

GOUVEIA, Andréa Barbosa Gouveia; FERRAZ, Marcos Alexandre dos Santos. Financiamento da educação e luta sindical: conflitos em uma grande rede de ensino. Educação \& Sociedade, Campinas, v. 37, n. 134, p. 285-302, jan.-mar. 2016. Disponível em: https://www.scielo.br/j/es/a/mvMXV3kDKSmkqbWBq3SvQLL/?lang=pt. Acesso em: 26 set. 2021. 
GOUVEIA, Andréa Barbosa; FERNANDES, Maria Dilneia Espíndola. Agenda Sindical de Professores da Educação Básica: desafios postos a partir do Piso Salarial Profissional Nacional. Práxis Educativa, Ponta Grossa, v. 14, n. 1, p. 84-98, jan./abr. 2019.

Disponível em: http://www.revistas2.uepg.br/index.php/praxiseducativa. Acesso em: 26 set. 2021.

JACOMINI; Márcia Aparecida; PENNA, Marieta Gouvêa de Oliveira Penna. Carreira docente e valorização do magistério: condições de trabalho e desenvolvimento profissional. Pro-posições, v. 27, n. 2, p. 177-202, maio/ago. 2016.

LIMA, Maria José Rocha. A trama da ignorância e outros escritos. Salvador: BDA, 1996.

NASCIMENTO, Ana Paula Santiago do; AMORIM, Renata Rodrigues de; CAMARGO, Rubens Barbosa de. Composição salarial dos docentes da Rede Estadual Paulista: uma análise a partir do Boletim de Acompanhamento de Pessoal da SEE/SP, 1996/2010. Fineduca - Revista de Financiamento da Educação, Porto Alegre, v. 4, n. 7, 2014.

OLIVEIRA, Dalila Andrade. As políticas de formação e a crise da profissionalização docente: por onde passa a valorização? Educação em Questão, Natal, v. 46, n. 32 , p. $51-74,2013$.

RODRIGUES, Robson da Silva; COSTA, Áurea de Carvalho. Da Constituição Federal de 1988 ao Plano Nacional de Educação 2014-2024: ardilosas apropriações da noção de valorização do trabalho docente. Política \& Trabalho - Revista de Ciências Sociais, n. 50, p. 231-248, jan./jun. 2019.

TEIXEIRA, Eliara Cristina Nogueira da Silva; NUNES, Cláudio Pinto Nunes. O piso salarial como insumo da valorização docente nos governos de FHC e Lula: da Política de Fundos à Lei do Piso. Práxis Educacional, v. 12, p. 251-270, 2016. 


\section{ANNEXE - NOTE DE BAS DE PAGE DE RÉFÉRENCE}

3. Cet article s'inscrit dans le cadre des travaux de recherche menés pour la thèse de doctorat en éducation intitulée Partidos Políticos e Piso Salarial do Magistério Baiano: no Discurso e na Prática, qui est en phase finale de préparation et sera défendue à l'Université internationale ibéro-américaine (UNINI).

4. Voir la note de bas de page numéro un.

5. Cf. note de bas de page $n^{\circ} 1$.

Soumis: Octobre, 2021.

Approbation: Novembre 2021. 